Supplement of Clim. Past, 16, 973-1006, 2020

https://doi.org/10.5194/cp-16-973-2020-supplement

(C) Author(s) 2020. This work is distributed under

the Creative Commons Attribution 4.0 License.

(c) (1)

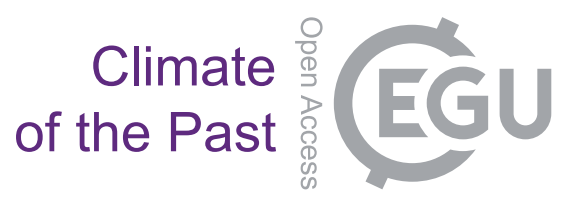

Supplement of

\title{
Paleogeographic controls on the evolution of Late Cretaceous ocean circulation
}

\section{Jean-Baptiste Ladant et al.}

Correspondence to: Jean-Baptiste Ladant (jbladant@umich.edu)

The copyright of individual parts of the supplement might differ from the CC BY 4.0 License. 
Water transport (Sv)

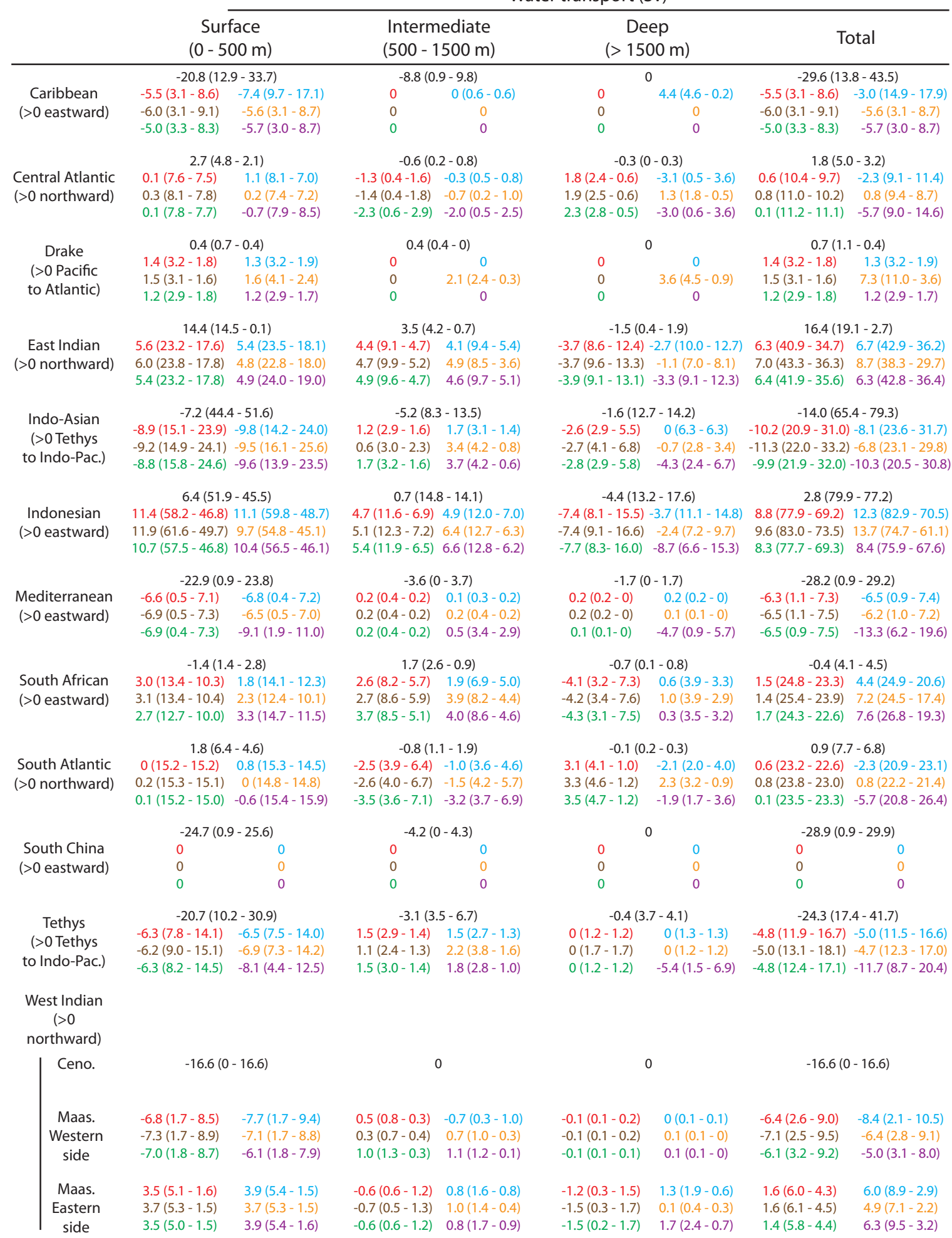

Deep Caribbean Seaway

Deep Drake Passage

Deep Neotethys Seaway

Table S1. Water transport across major oceanic sections (shown on Figs. 5 and 6) for each simulation. For each section is shown the direction of the positive transport across the gateway. There is 
bidirectional flow, i.e. positive and negative water fluxes, across most sections. The sum of positive and negative fluxes across a section gives the net water transport across the section. The net water transport is shown for three depth ranges (upper, intermediate and deep ocean) and for the total vertical extension of the section. The summed positive and negative fluxes for the depth intervals considered are also shown in parentheses. The sign of the net water transport therefore gives the direction of the larger water flux across a section. Positive and negative fluxes are represented on Figs. 5 and 6 for the intermediate and deep ocean respectively.

\begin{tabular}{cccccccccc} 
& \multicolumn{3}{c}{ Cenomanian } & \multicolumn{3}{c}{ Maastrichtian } \\
\hline $\begin{array}{c}\text { Total runoff supply }(\mathrm{mSv}) \\
\text { Total basin area }\left(10^{6} \mathrm{~km}^{2}\right)\end{array}$ & Pacific & Atlantic & $\begin{array}{c}\text { Indo-Neo- } \\
\text { tethys }\end{array}$ & $\begin{array}{c}\text { Indo-Atlantic- } \\
\text { Neotethys }\end{array}$ & Pacific & Atlantic & $\begin{array}{c}\text { Indo-Neo- } \\
\text { tethys }\end{array}$ & $\begin{array}{c}\text { Indo-Atlantic- } \\
\text { Neotethys }\end{array}$ \\
Global & $\frac{833}{21.84}$ & $\frac{654}{46.46}$ & $\frac{945}{100.57}$ & $\frac{1599}{147.03}$ & $\frac{792}{212.27}$ & $\frac{864}{58.97}$ & $\frac{931}{94.96}$ & $\frac{1796}{153.93}$ \\
$\begin{array}{c}\text { Southern Hemisphere } \\
\text { high-latitudes }\end{array}$ & $\frac{130}{19.95}$ & $\frac{54}{5.59}$ & $\frac{195}{8.03}$ & $\frac{248}{13.63}$ & $\frac{129}{17.61}$ & $\frac{55}{9.27}$ & $\frac{211}{11.11}$ & $\frac{266}{20.38}$ \\
$\begin{array}{c}\text { Northern Hemisphere } \\
\text { high-latitudes }\end{array}$ & $\frac{130}{15.49}$ & $\frac{175}{6.79}$ & $\frac{72}{1.37}$ & $\frac{248}{8.15}$ & $\frac{169}{15.04}$ & $\frac{160}{3.53}$ & $\frac{47}{1.75}$ & $\frac{207}{5.28}$ \\
$\begin{array}{c}\text { Mid- and low- } \\
\text { latitudes }\end{array}$ & $\frac{573}{186.4}$ & $\frac{425}{34.08}$ & $\frac{678}{91.17}$ & $\frac{1103}{125.25}$ & $\frac{494}{179.62}$ & $\frac{649}{46.18}$ & $\frac{674}{82.1}$ & $\frac{1323}{28.28}$
\end{tabular}

Table S2. Total runoff freshwater supply $(\mathrm{mSv})$ for the Cenomanian and Maastrichtian simulations. The Pacific, Atlantic and Indo-Neotethyan basins are defined as shown on Fig. S3. Southern and northern high-latitudes are defined as latitudes $<50^{\circ} \mathrm{S}$ and $>50^{\circ} \mathrm{N}$ respectively. 


\begin{tabular}{|c|c|c|c|c|c|c|}
\hline Site & Location & Paleolatitude & Paleolongitude & $\begin{array}{l}\text { Paleodepth } \\
\text { (m) }\end{array}$ & $\begin{array}{c}\text { Average } \\
\varepsilon_{\mathrm{Nd}} \\
\end{array}$ & References \\
\hline 765 & $\begin{array}{c}\text { Argo Abyssal } \\
\text { Plain }\end{array}$ & $40^{\circ} \mathrm{S}$ & $105^{\circ} \mathrm{E}$ & 5000 & -7.9 & MT12, GL92 \\
\hline 763 & $\begin{array}{l}\text { Central } \\
\text { Exmouth } \\
\text { Plateau }\end{array}$ & $44^{\circ} \mathrm{S}$ & $100^{\circ} \mathrm{E}$ & 500 & -9.8 & $\begin{array}{l}\text { LH12, MT12, } \\
\text { V13, GL92 }\end{array}$ \\
\hline 766 & $\begin{array}{c}\text { Gascoyne } \\
\text { Abyssal Plain }\end{array}$ & $45^{\circ} \mathrm{S}$ & $95^{\circ} \mathrm{E}$ & 3000 & -6.8 & $\begin{array}{l}\text { R10, MT12, } \\
\text { GL92 }\end{array}$ \\
\hline 258 & $\begin{array}{l}\text { Naturaliste } \\
\text { Plateau }\end{array}$ & $57^{\circ} \mathrm{S}$ & $85^{\circ} \mathrm{E}$ & $2500-3000$ & -8.0 & M16 \\
\hline 1135 & $\begin{array}{l}\text { Southern } \\
\text { Kerguelen } \\
\text { Plateau }\end{array}$ & $55^{\circ} \mathrm{S}$ & $72^{\circ} \mathrm{E}$ & $1300-2000$ & -7.9 & LH12 \\
\hline 1138 & $\begin{array}{l}\text { Kerguelen } \\
\text { Plateau }\end{array}$ & $52^{\circ} \mathrm{S}$ & $70^{\circ} \mathrm{E}$ & $500-1500$ & -5.6 & MT12 \\
\hline 551 & Goban Spur & $40^{\circ} \mathrm{N}$ & $2^{\circ} \mathrm{W}$ & 1500 & -7.1 & M12, M85 \\
\hline 530 & $\begin{array}{c}\text { SE Angola } \\
\text { Basin }\end{array}$ & $30^{\circ} \mathrm{S}$ & $4^{\circ} \mathrm{W}$ & $>2000$ & -7.5 & $\mathrm{R} 10$ \\
\hline 530 & $\begin{array}{c}\text { SE Angola } \\
\text { Basin }\end{array}$ & $31^{\circ} \mathrm{S}$ & $5^{\circ} \mathrm{W}$ & 3000 & -7.7 & MT13 \\
\hline 361 & Cape Basin & $45^{\circ} \mathrm{S}$ & $5^{\circ} \mathrm{W}$ & 4000 & -6.1 & MT13 \\
\hline 367 & Cape Verde & $17^{\circ} \mathrm{N}$ & $18^{\circ} \mathrm{W}$ & $>2000$ & -9.0 & M12 \\
\hline 700 & $\begin{array}{c}\text { East Georgia } \\
\text { Basin }\end{array}$ & $52^{\circ} \mathrm{S}$ & $18^{\circ} \mathrm{W}$ & $1500-2000$ & -7.7 & M16 \\
\hline 1276 & $\begin{array}{l}\text { Newfoundland } \\
\text { Margin }\end{array}$ & $35^{\circ} \mathrm{N}$ & $22^{\circ} \mathrm{W}$ & $>2000$ & -6.8 & RV12 \\
\hline 511 & $\begin{array}{c}\text { Falkland } \\
\text { Plateau }\end{array}$ & $54^{\circ} \mathrm{S}$ & $25^{\circ} \mathrm{W}$ & $>1000$ & -5.2 & R10 \\
\hline 511 & $\begin{array}{c}\text { Falkland } \\
\text { Basin }\end{array}$ & $52^{\circ} \mathrm{S}$ & $26^{\circ} \mathrm{W}$ & 1800 & -5.1 & MT13 \\
\hline 1261 & $\begin{array}{c}\text { Demerara } \\
\text { Rise } \\
\end{array}$ & $6^{\circ} \mathrm{N}$ & $29^{\circ} \mathrm{W}$ & $1000-1500$ & -13.0 & M12 \\
\hline 1260 & $\begin{array}{l}\text { Demerara } \\
\text { Rise }\end{array}$ & $8^{\circ} \mathrm{N}$ & $31^{\circ} \mathrm{W}$ & $1000-1500$ & -13.5 & $\begin{array}{c}\text { ML08, } \\
\text { ML11, M12, } \\
\text { JB10 }\end{array}$ \\
\hline 1258 & $\begin{array}{l}\text { Demerara } \\
\text { Rise }\end{array}$ & $9^{\circ} \mathrm{N}$ & $32^{\circ} \mathrm{W}$ & $1000-1500$ & -13.5 & $\begin{array}{c}\text { ML08, } \\
\text { ML11, M12, } \\
\text { JB10 }\end{array}$ \\
\hline 386 & Bermuda Rise & $27^{\circ} \mathrm{N}$ & $35^{\circ} \mathrm{W}$ & $>2000$ & -6.8 & M12, RV12 \\
\hline 1050 & Blake Nose & $26^{\circ} \mathrm{N}$ & $42^{\circ} \mathrm{W}$ & $1000-2000$ & -5.2 & M12 \\
\hline 1208 & Shatsky Rise & $15^{\circ} \mathrm{N}$ & $154^{\circ} \mathrm{W}$ & $1500-2000$ & -3.4 & MT12 \\
\hline
\end{tabular}

Table S3. Cenomanian $\varepsilon_{\mathrm{Nd}}$ compilation based on Moiroud et al. (2016). The $\varepsilon_{\mathrm{Nd}}$ values are averaged between $100 \mathrm{Ma}$ and $90 \mathrm{Ma}$ (see main text). GL92: Gradstein and Ludden (1992), JB10: Jiménez Berrocoso et al. (2010), LH12: Le Houedec et al. (2012), M12: Martin et al. (2012), M16: Moiroud et al. (2016), M85: Masson et al. (1985), ML08: MacLeod et al. (2008), ML11: MacLeod et al. (2011), MT12: Murphy and Thomas (2012), MT13: Murphy and Thomas (2013), R10: Robinson et al. (2010), RV12: Robinson and Vance (2012), V13: Voigt et al. (2013). 


\begin{tabular}{|c|c|c|c|c|c|c|}
\hline Site & Location & Paleolatitude & Paleolongitude & $\begin{array}{l}\text { Paleodepth } \\
(\mathrm{m})\end{array}$ & $\begin{array}{c}\text { Average } \\
\varepsilon_{\mathrm{Nd}} \\
\end{array}$ & References \\
\hline 765 & $\begin{array}{c}\text { Argo Abyssal } \\
\text { Plain }\end{array}$ & $40^{\circ} \mathrm{S}$ & $105^{\circ} \mathrm{E}$ & 5000 & -10.2 & MT12, GL92 \\
\hline 762 & $\begin{array}{l}\text { Central } \\
\text { Exmouth } \\
\text { Plateau }\end{array}$ & $44^{\circ} \mathrm{S}$ & $100^{\circ} \mathrm{E}$ & 750 & -11.0 & $\begin{array}{l}\text { LH12, V13, } \\
\text { GL92 }\end{array}$ \\
\hline 766 & $\begin{array}{c}\text { Gascoyne } \\
\text { Abyssal Plain }\end{array}$ & $46^{\circ} \mathrm{S}$ & $98^{\circ} \mathrm{E}$ & 3000 & -9.4 & $\begin{array}{l}\text { R10, MT12, } \\
\text { GL92 }\end{array}$ \\
\hline 1135 & $\begin{array}{c}\text { Southern } \\
\text { Kerguelen } \\
\text { Plateau } \\
\end{array}$ & $58^{\circ} \mathrm{S}$ & $72^{\circ} \mathrm{E}$ & $1300-2000$ & -9.6 & LH12 \\
\hline 1138 & $\begin{array}{c}\text { Kerguelen } \\
\text { Plateau }\end{array}$ & $55^{\circ} \mathrm{S}$ & $70^{\circ} \mathrm{E}$ & $500-1500$ & -8.5 & MT12 \\
\hline 758 & $\begin{array}{l}\text { Ninetyeast } \\
\text { Ridge }\end{array}$ & $47^{\circ} \mathrm{S}$ & $50^{\circ} \mathrm{E}$ & $1500-2000$ & -10.3 & LH12 \\
\hline 530 & $\begin{array}{c}\text { SE Angola } \\
\text { Basin }\end{array}$ & $30^{\circ} \mathrm{S}$ & $1^{\circ} \mathrm{W}$ & $>2000$ & -10.0 & $\mathrm{R} 10$ \\
\hline 551 & Goban Spur & $37^{\circ} \mathrm{N}$ & $5^{\circ} \mathrm{W}$ & 2100 & -9.9 & M12, M85 \\
\hline 690 & Maud Rise & $65^{\circ} \mathrm{S}$ & $6^{\circ} \mathrm{W}$ & 1800 & -9.8 & $\mathrm{~V} 13$ \\
\hline 525 & Walvis Ridge & $36^{\circ} \mathrm{S}$ & $8^{\circ} \mathrm{W}$ & $1000-1500$ & -3.5 & V13 \\
\hline 367 & Cape Verde & $15^{\circ} \mathrm{N}$ & $20^{\circ} \mathrm{W}$ & $>2000$ & -13.4 & M12 \\
\hline 700 & $\begin{array}{l}\text { East Georgia } \\
\text { Basin }\end{array}$ & $52^{\circ} \mathrm{S}$ & $20^{\circ} \mathrm{W}$ & $1500-2000$ & -8.2 & M16 \\
\hline 1276 & $\begin{array}{l}\text { Newfoundland } \\
\text { Margin }\end{array}$ & $35^{\circ} \mathrm{N}$ & $22^{\circ} \mathrm{W}$ & $>2000$ & -6.6 & RV12 \\
\hline 357 & $\begin{array}{l}\text { Rio Grande } \\
\text { Rise }\end{array}$ & $34^{\circ} \mathrm{S}$ & $25^{\circ} \mathrm{W}$ & 1500 & -9.7 & MT13 \\
\hline 511 & $\begin{array}{c}\text { Falkland } \\
\text { Plateau }\end{array}$ & $54^{\circ} \mathrm{S}$ & $29^{\circ} \mathrm{W}$ & $>1000$ & -8.7 & $\mathrm{R} 10$ \\
\hline 1261 & Demerara Rise & $3^{\circ} \mathrm{N}$ & $29^{\circ} \mathrm{W}$ & $1000-1500$ & -15.9 & ML11, M12 \\
\hline 1260 & Demerara Rise & $5^{\circ} \mathrm{N}$ & $31^{\circ} \mathrm{W}$ & $1000-1500$ & -15.2 & $\begin{array}{c}\text { ML08, } \\
\text { ML11, M12 }\end{array}$ \\
\hline 1258 & Demerara Rise & $6^{\circ} \mathrm{N}$ & $32^{\circ} \mathrm{W}$ & $1000-1500$ & -14.8 & $\begin{array}{c}\text { ML08, } \\
\text { ML11, M12 }\end{array}$ \\
\hline 386 & Bermuda Rise & $26^{\circ} \mathrm{N}$ & $40^{\circ} \mathrm{W}$ & $>2000$ & -9.5 & M12, RV12 \\
\hline 1050 & Blake Nose & $30^{\circ} \mathrm{N}$ & $54^{\circ} \mathrm{W}$ & $1000-2000$ & -8.5 & M12 \\
\hline 152 & $\begin{array}{c}\text { Nicaraguan } \\
\text { Rise }\end{array}$ & $15^{\circ} \mathrm{N}$ & $70^{\circ} \mathrm{W}$ & $1500-2000$ & -5.7 & M16 \\
\hline 323 & $\begin{array}{c}\text { Bellingshausen } \\
\text { Plateau }\end{array}$ & $67^{\circ} \mathrm{S}$ & $100^{\circ} \mathrm{W}$ & 2000 & -3.8 & $\mathrm{~T} 14$ \\
\hline 596 & South Pacific & $45^{\circ} \mathrm{S}$ & $120^{\circ} \mathrm{W}$ & 5000 & -5.6 & T14 \\
\hline 1186 & $\begin{array}{l}\text { Ontong-Java } \\
\text { Plateau }\end{array}$ & $15^{\circ} \mathrm{S}$ & $132^{\circ} \mathrm{W}$ & 2800 & -5.1 & $\mathrm{H} 20$ \\
\hline 465 & $\begin{array}{c}\text { Southern Hess } \\
\text { Rise }\end{array}$ & $8^{\circ} \mathrm{N}$ & $138^{\circ} \mathrm{W}$ & 900 & -3.8 & $\mathrm{H} 12$ \\
\hline 464 & $\begin{array}{c}\text { Northern Hess } \\
\text { Rise }\end{array}$ & $12^{\circ} \mathrm{N}$ & $140^{\circ} \mathrm{W}$ & 4000 & -5.1 & H12 \\
\hline 883 & $\begin{array}{c}\text { Detroit } \\
\text { Seamount } \\
\end{array}$ & $34^{\circ} \mathrm{N}$ & $140^{\circ} \mathrm{W}$ & 2000 & 2.4 & H12, DT04 \\
\hline 886 & $\begin{array}{l}\text { Chinook } \\
\text { Trough }\end{array}$ & $30^{\circ} \mathrm{N}$ & $140^{\circ} \mathrm{W}$ & $>4400$ & -4.2 & ML08 \\
\hline 463 & $\begin{array}{l}\text { Mid-Pacific } \\
\text { Mountains }\end{array}$ & $5^{\circ} \mathrm{S}$ & $145^{\circ} \mathrm{W}$ & $1500-2000$ & -5.0 & $\mathrm{H} 20$ \\
\hline
\end{tabular}




\begin{tabular}{c|l|c|c|c|c|c}
\hline 1208 & Shatsky Rise & $15^{\circ} \mathrm{N}$ & $154^{\circ} \mathrm{W}$ & 3300 & -4.0 & $\mathrm{H} 12$ \\
\hline 1209 & Shatsky Rise & $12^{\circ} \mathrm{N}$ & $158^{\circ} \mathrm{W}$ & $2000-3000$ & -4.3 & T04, F05 \\
\hline 1210 & Shatsky Rise & $10^{\circ} \mathrm{N}$ & $160^{\circ} \mathrm{W}$ & $2000-3000$ & -4.3 & F05 \\
\hline 1211 & Shatsky Rise & $8^{\circ} \mathrm{N}$ & $162^{\circ} \mathrm{W}$ & 2900 & -4.2 & T04
\end{tabular}

Table S4. Maastrichtian $\varepsilon_{\mathrm{Nd}}$ compilation based on Moiroud et al. (2016). The $\varepsilon_{\mathrm{Nd}}$ values are averaged between $75 \mathrm{Ma}$ and $65 \mathrm{Ma}$ (see main text). DT04: Doubrovine and Tarduno (2004), F05: Frank et al. (2005), GL92: Gradstein and Ludden (1992), H12: Hague et al. (2012), H20: Haynes et al. (2020), LH12: Le Houedec et al. (2012), M12: Martin et al. (2012), M16: Moiroud et al. (2016), M85: Masson et al. (1985), ML08: MacLeod et al. (2008), ML11: MacLeod et al. (2011), MT12: Murphy and Thomas (2012), MT13: Murphy and Thomas (2013), R10: Robinson et al. (2010), RV12: Robinson and Vance (2012), T04: Thomas (2004), T14: Thomas et al. (2014), V13: Voigt et al. (2013). 

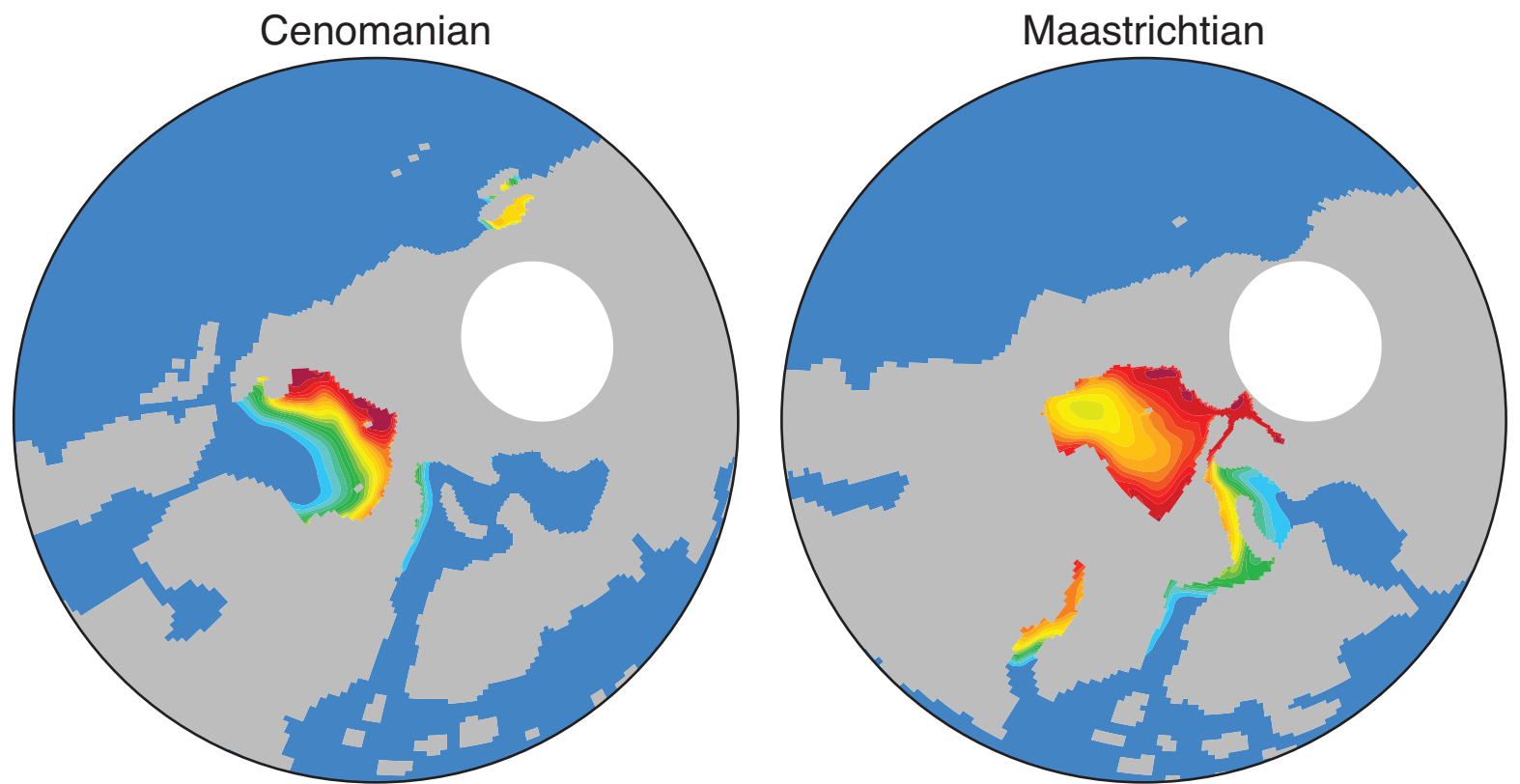

Winter sea ice fraction (\%)

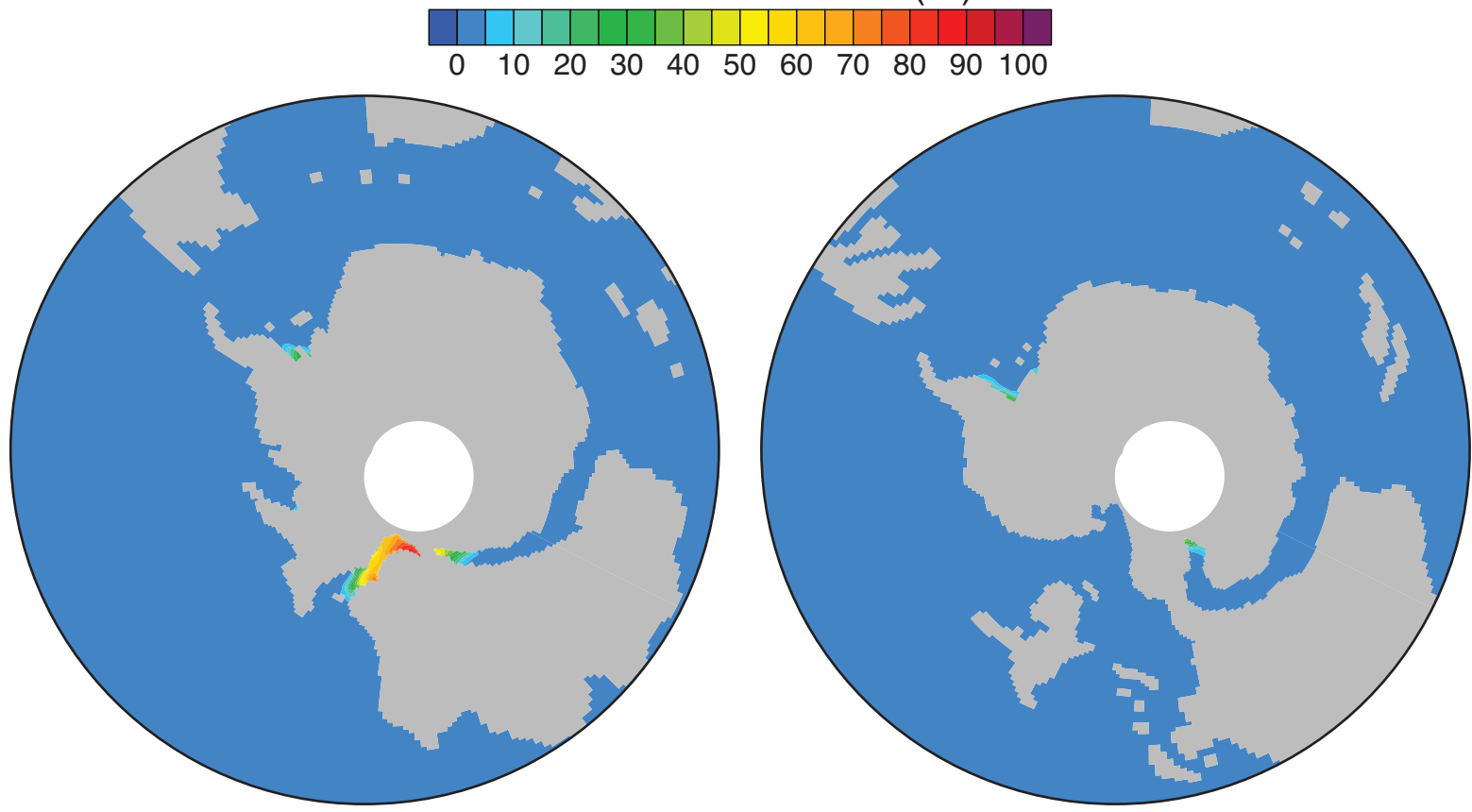

Figure S1. Mean winter sea ice fraction in the Cenomanian and Maastrichtian simulations. 

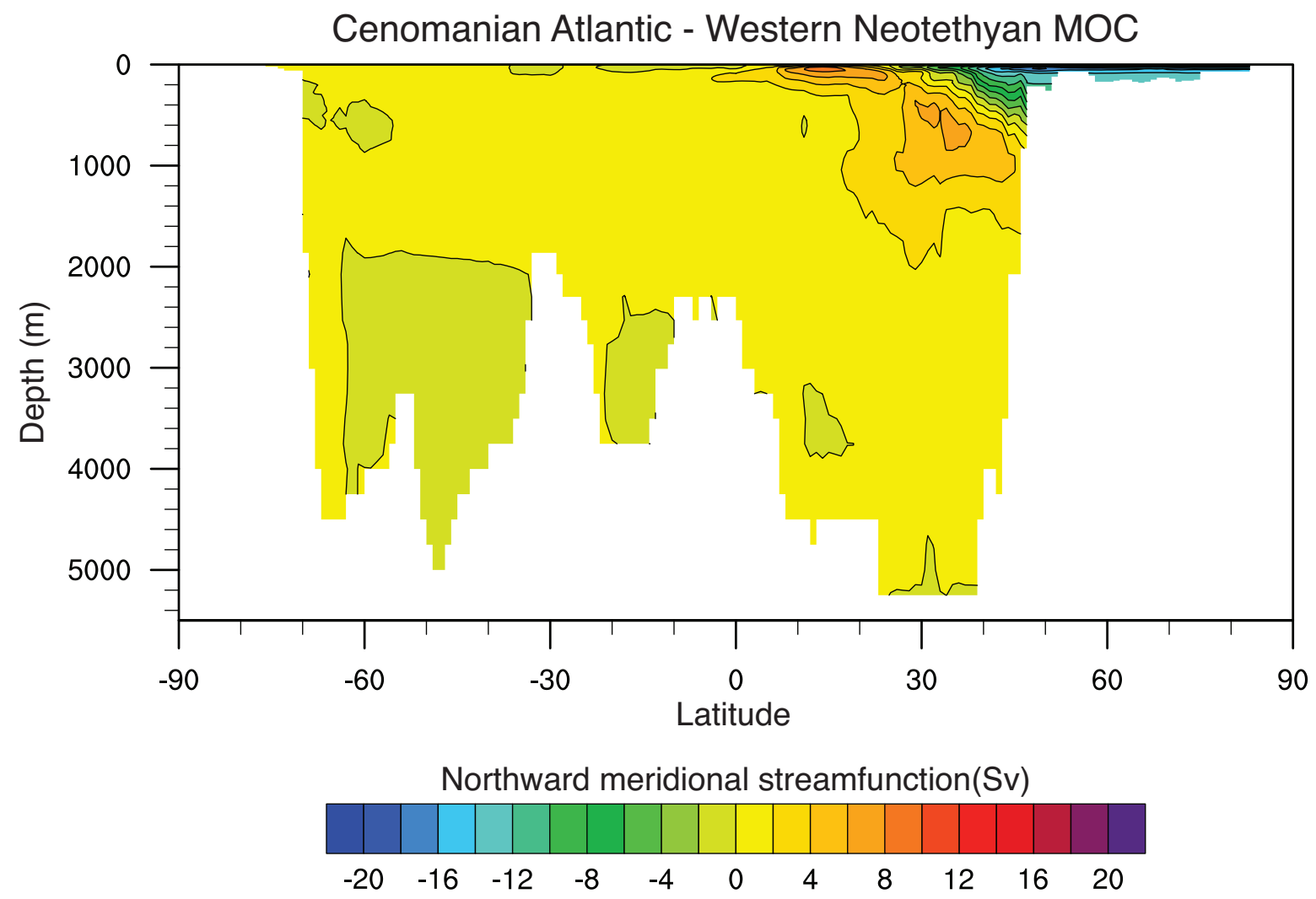

Figure S2. Meridional Overturning Circulation (Sv) in the Atlantic-Western Neotethyan basin of the Cenomanian simulation (Fig. S3).
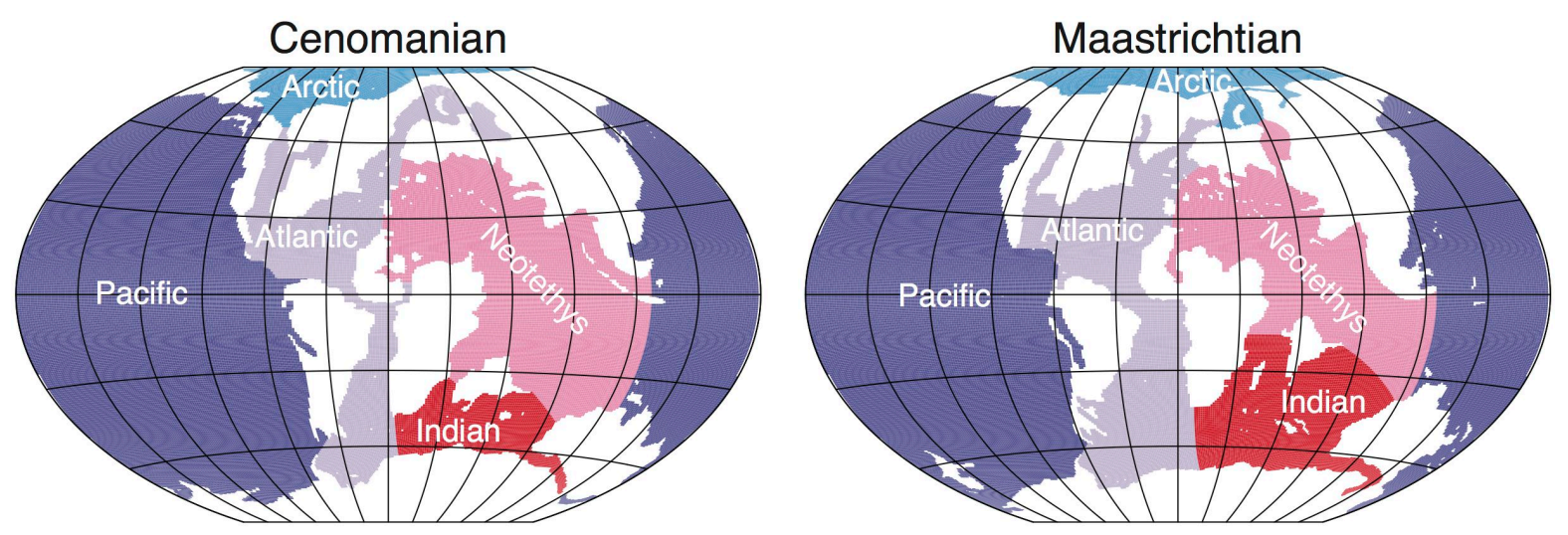

Figure S3. Configuration of the basin mask used in each simulation. In the text, the Neotethyan Ocean is sometimes split in a western Neotethyan Ocean (between Africa and Europe) and an eastern Neotethyan Ocean (between India and Asia). 


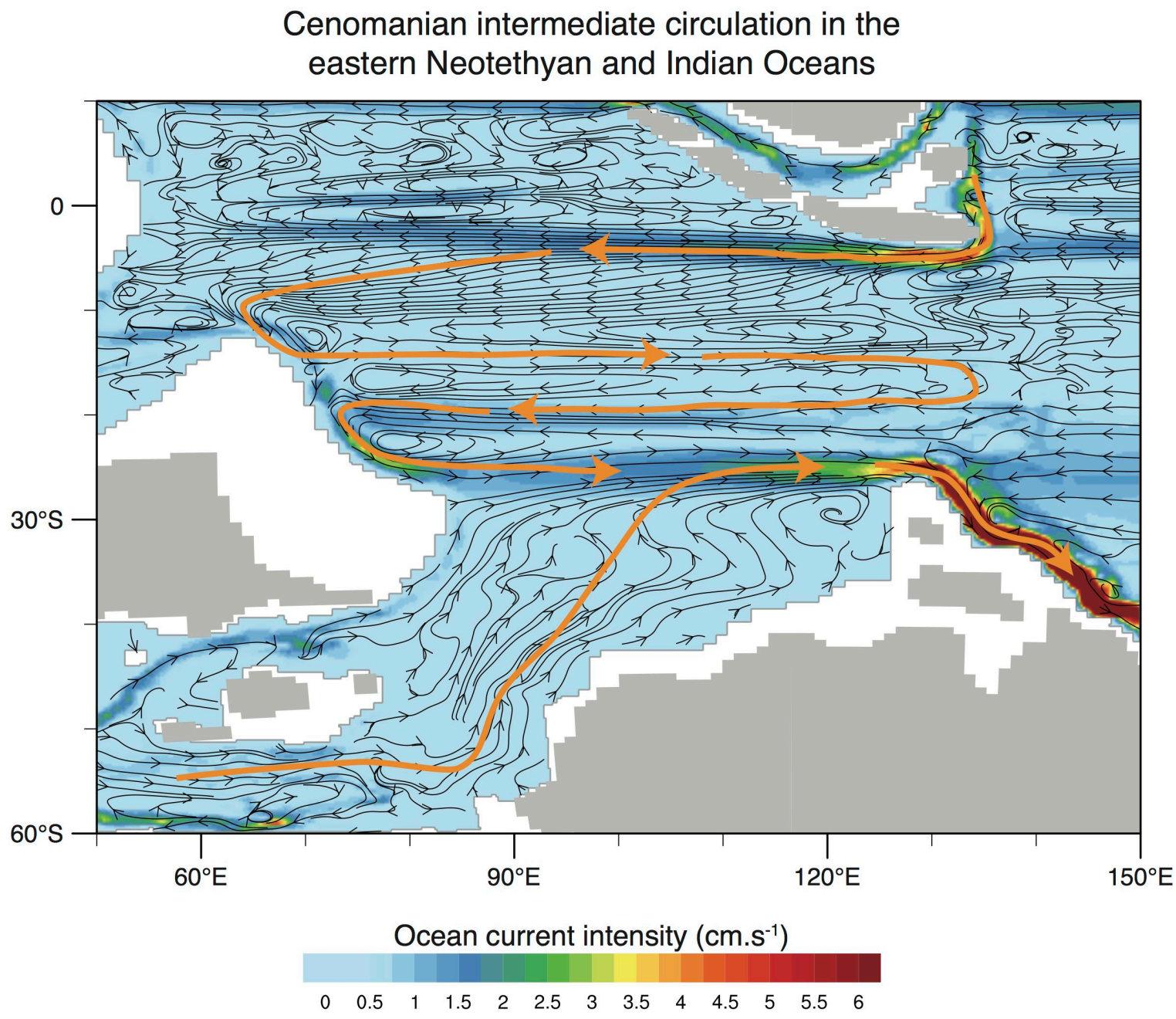

Figure S4. Cenomanian intermediate circulation $(1000 \mathrm{~m})$ in the eastern Neotethyan and Indian Oceans. Orange arrows represent major intermediate current systems in the eastern Neotethyan and Indian Oceans. 


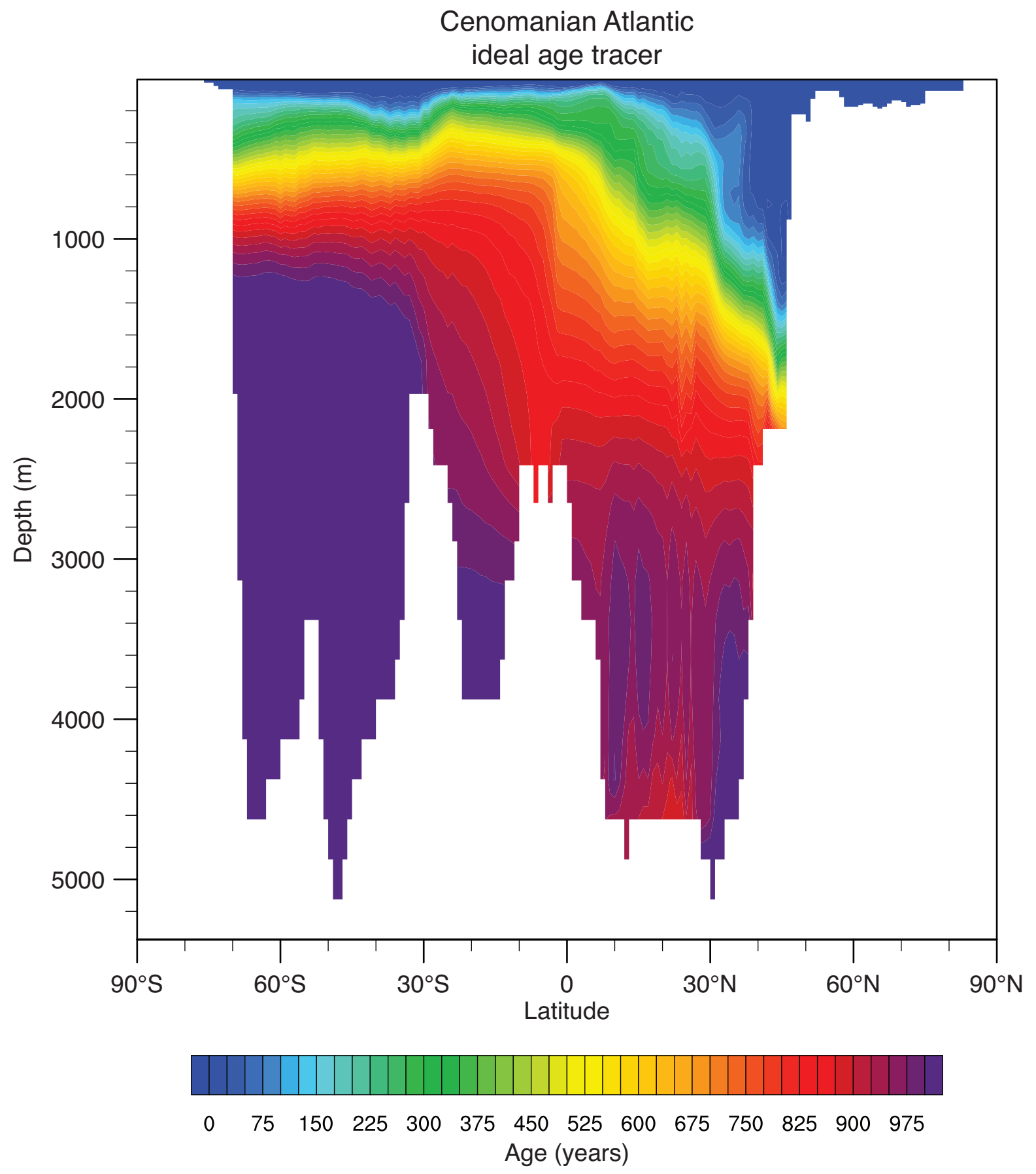

Figure S5. Cenomanian annual mean ideal age tracer in the Atlantic basin. 

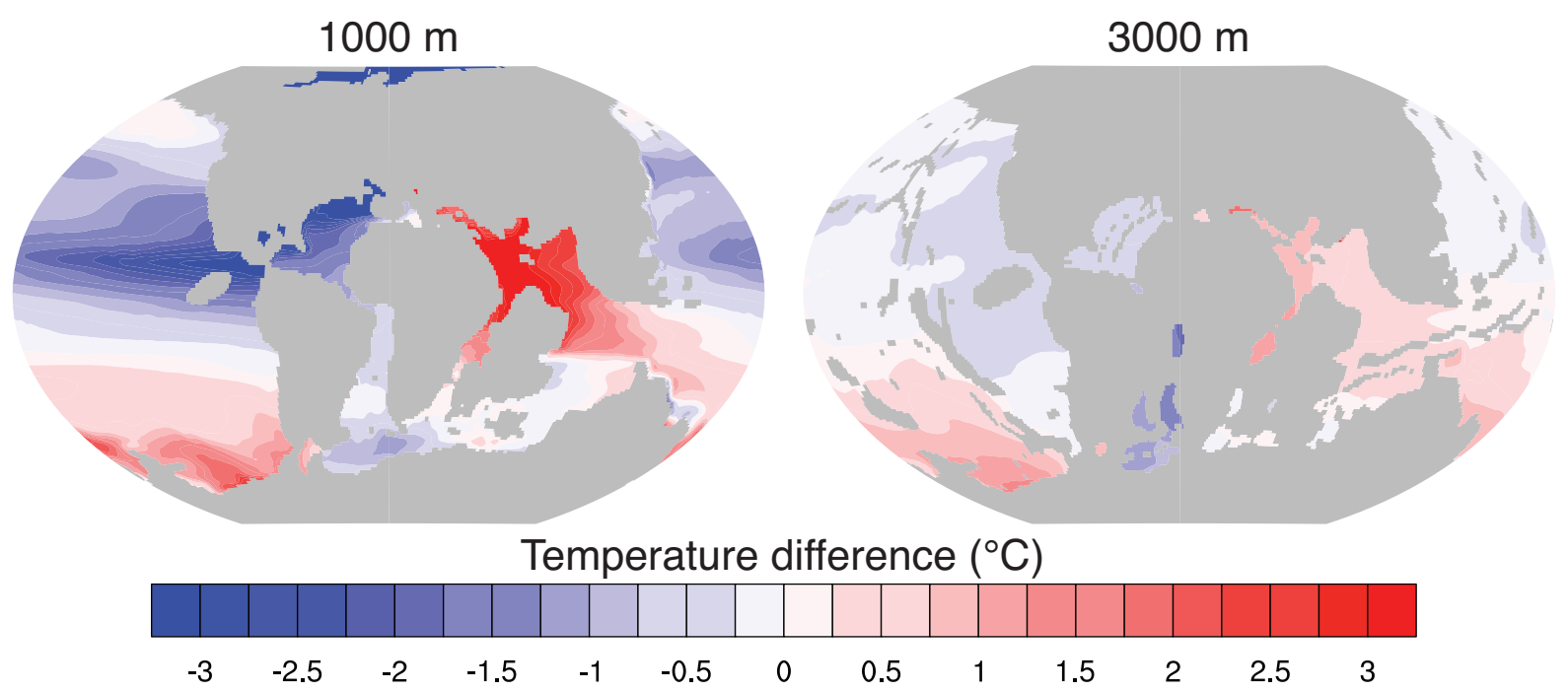

Figure S6. Intermediate $(\sim 1000 \mathrm{~m})$ and deep $(\sim 3000 \mathrm{~m})$ ocean temperature difference $\left({ }^{\circ} \mathrm{C}\right)$ between the Maastrichtian and the Cenomanian simulations. 

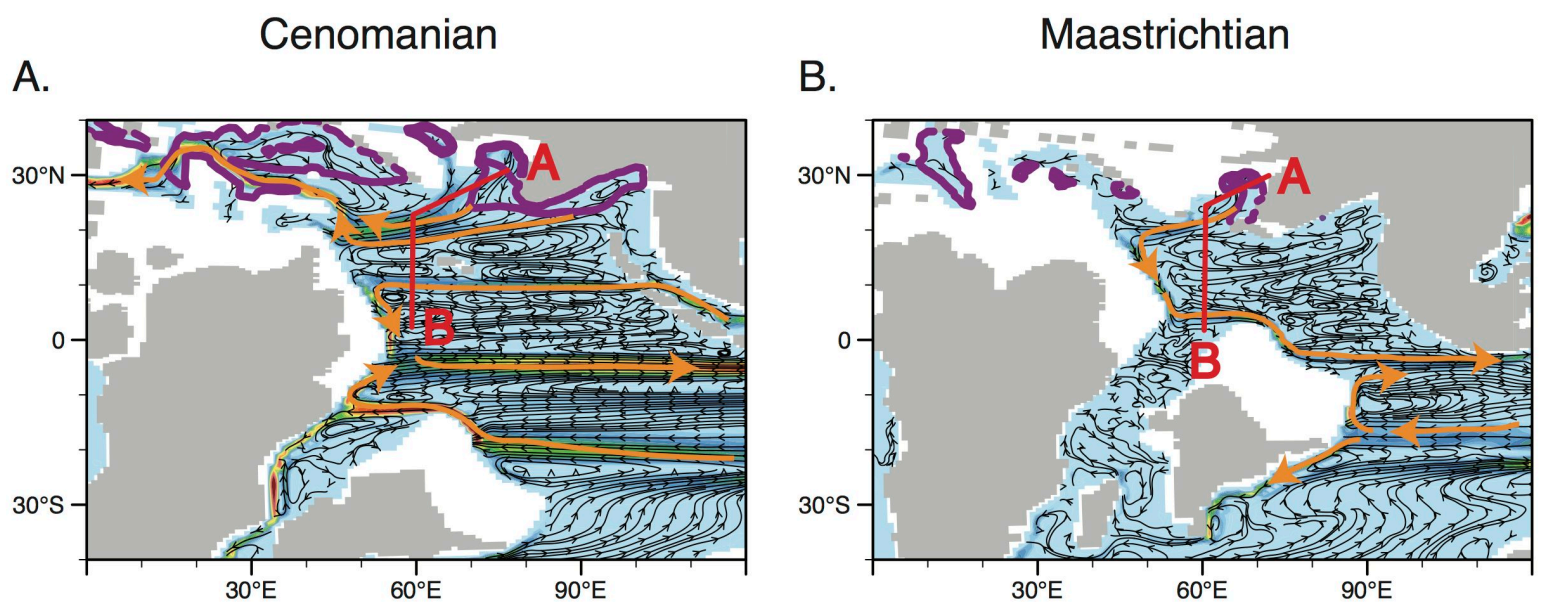

\section{$500 \mathrm{~m}$ depth ocean current intensity $\left(\mathrm{cm}^{\mathrm{s}} \mathrm{s}^{-1}\right)$}

\section{C.}

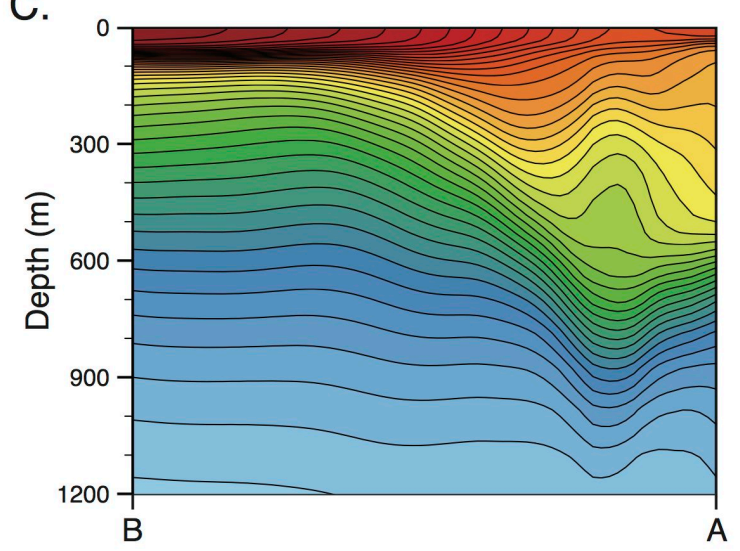

D.

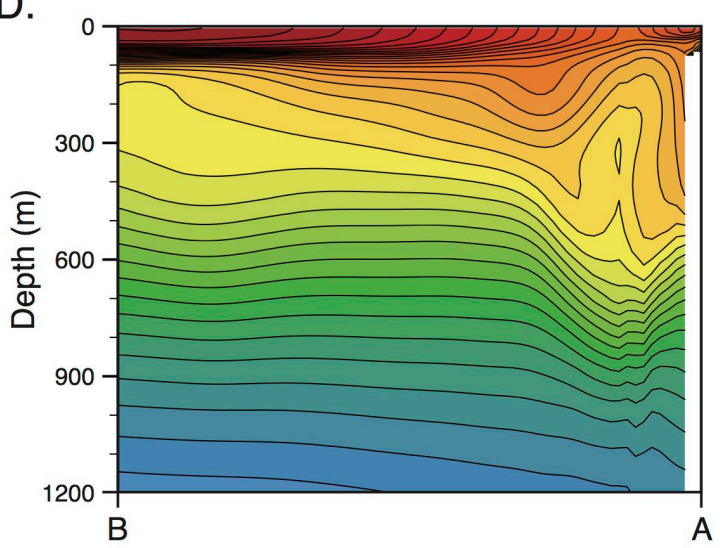

Temperature along $\mathrm{A}-\mathrm{B}$ transect $\left({ }^{\circ} \mathrm{C}\right)$

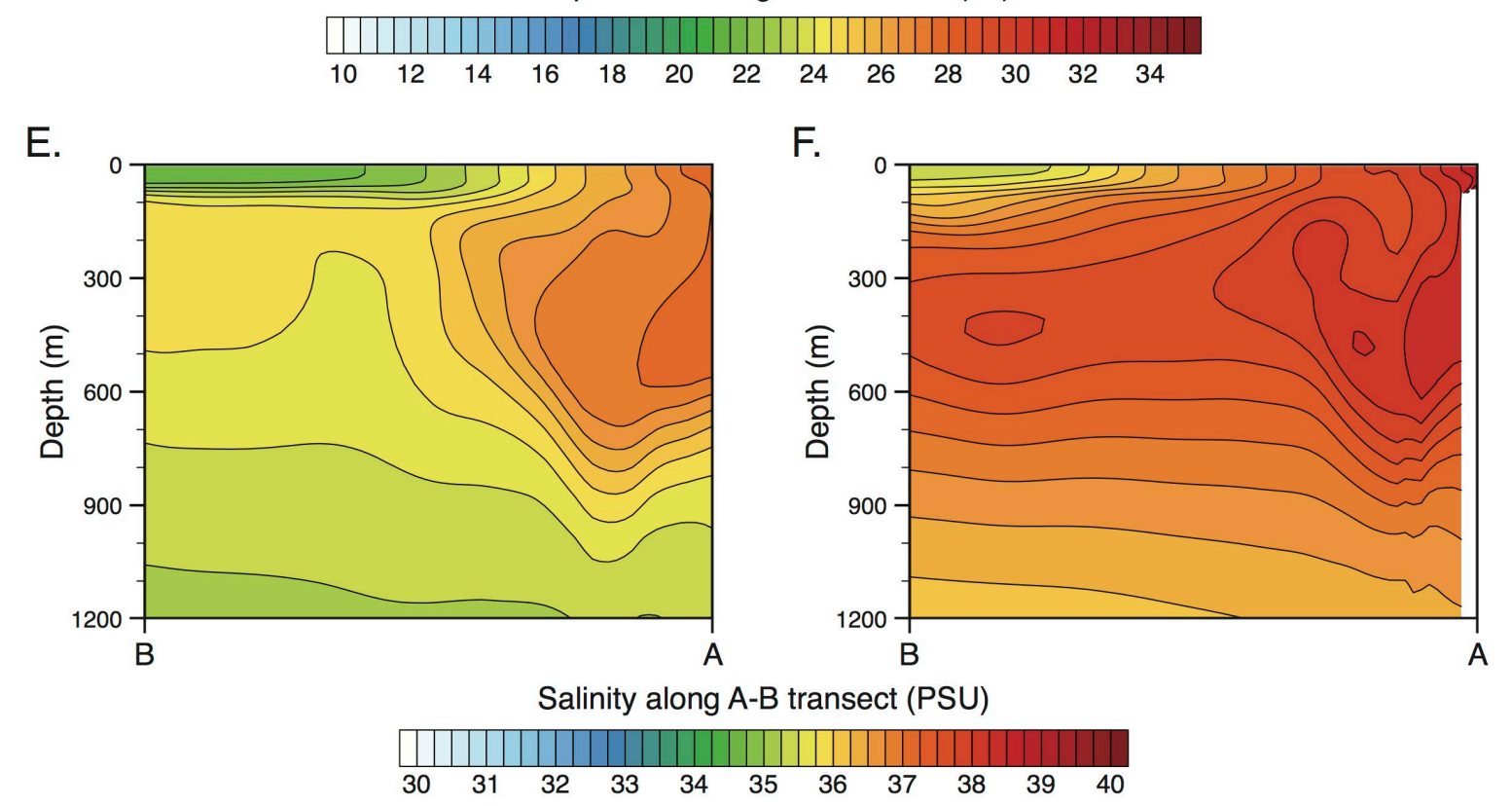

Figure S7. Upper intermediate $(\sim 500 \mathrm{~m})$ ocean circulation for the Cenomanian (A) and Maastrichtian (B) simulations. Orange arrows represent major current systems in the 
Neotethyan Ocean. Purple contours represent regions of late winter deepening of the mixed layer (200 m contours). Section A-B defines an ocean transect between regions of deeper winter MLD and the equatorial Neotethyan Ocean. (C and D) Ocean temperatures along the A-B transect for the Cenomanian and Maastrichtian respectively. (E and F) Same as C and D for the ocean salinity.

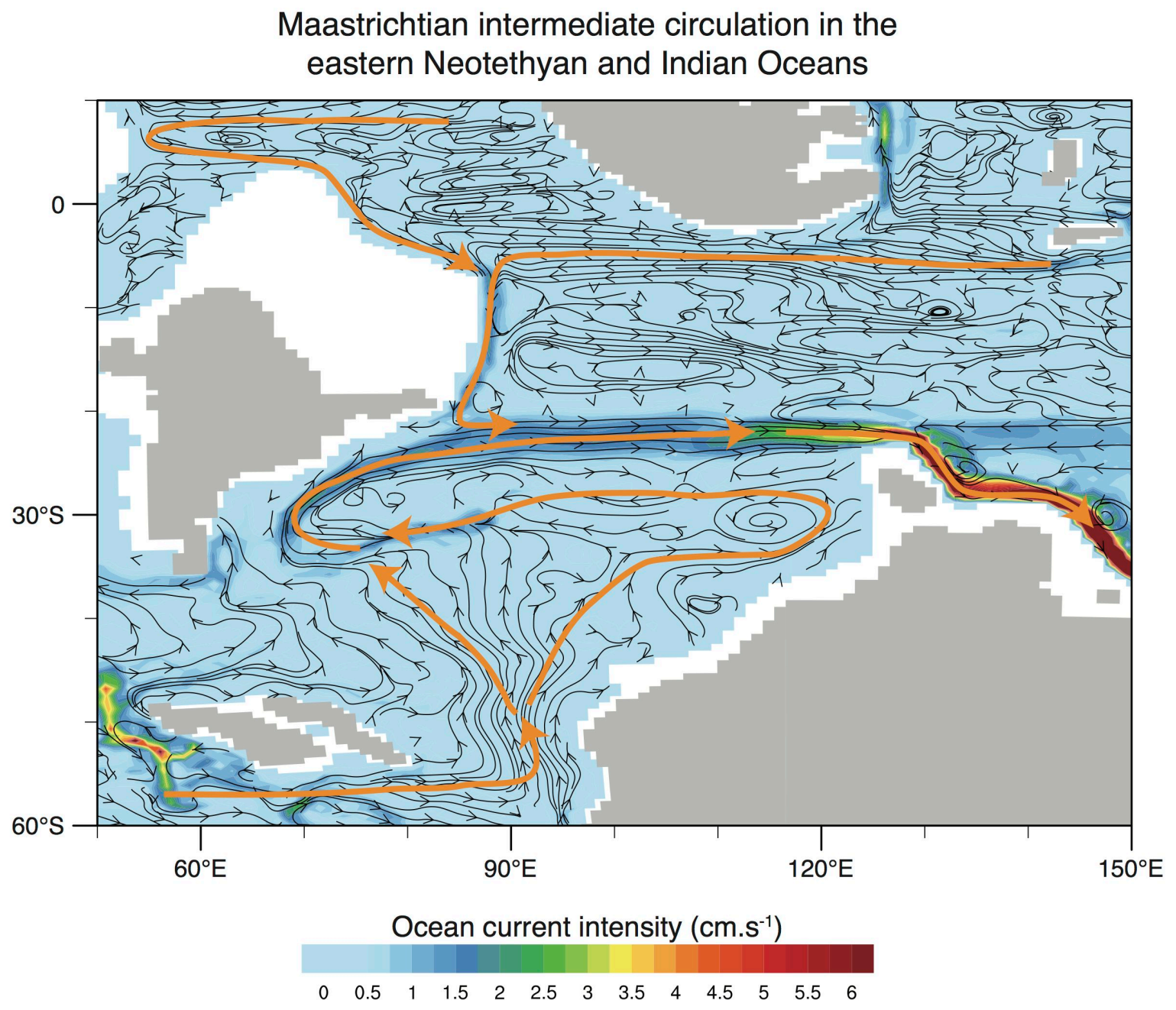

Figure S8. Maastrichtian intermediate circulation $(1000 \mathrm{~m})$ in the eastern Neotethyan and Indian Oceans. Orange arrows represent major intermediate current systems in the eastern Neotethyan and Indian Oceans. 
A. Deep Labrador Seaway - Maastrichtian

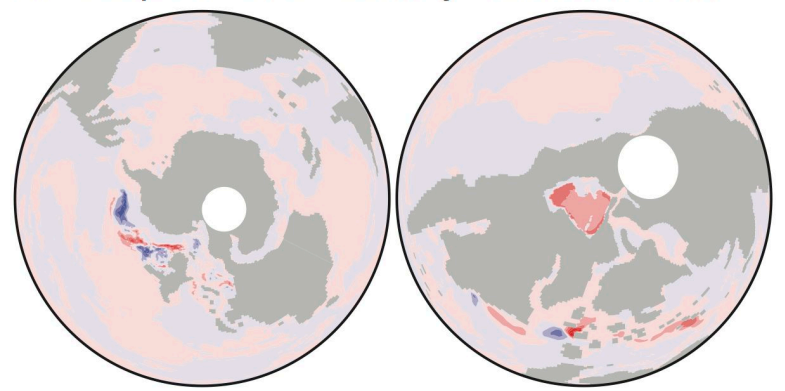

C. Deep Caribbean Seaway - Maastrichtian

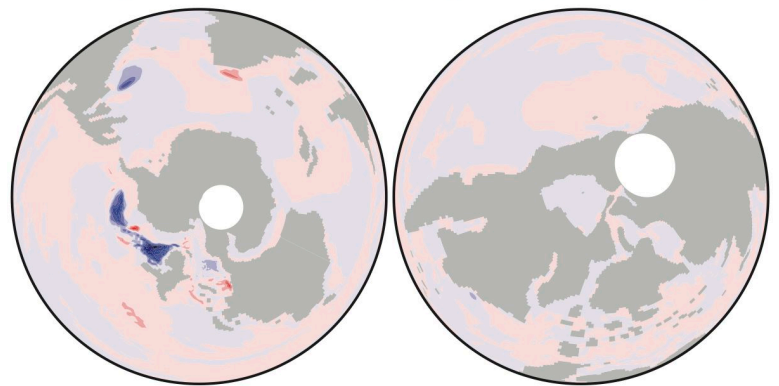

E. Maastrichtian $2 \times \mathrm{CO}_{2}$ - Maastrichtian

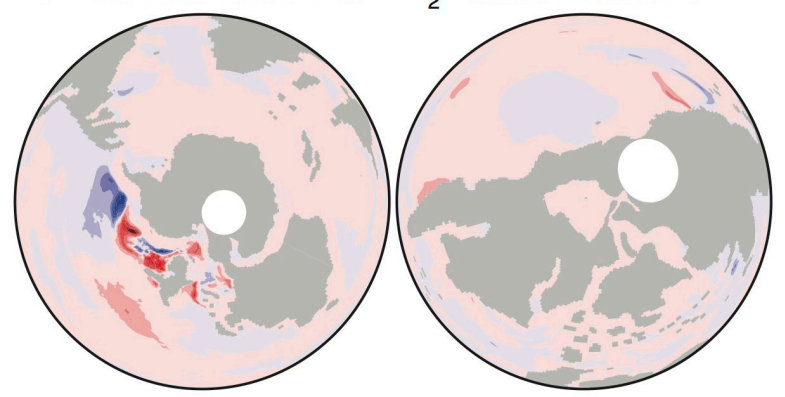

B. Deep Drake Passage - Maastrichtian

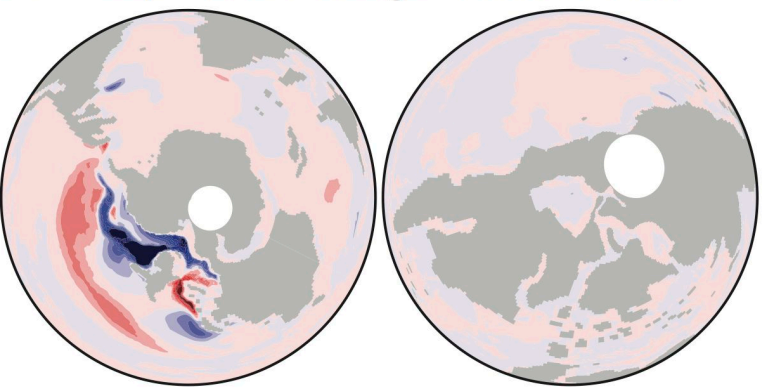

D. Deep Neotethys Seaway - Maastrichtian

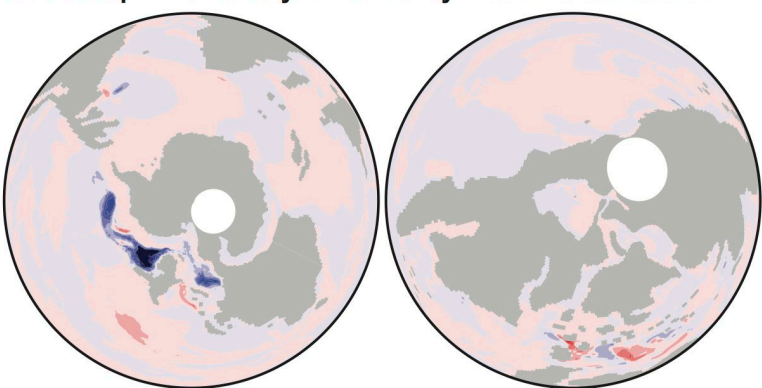

Late winter mixed layer depth difference (m)

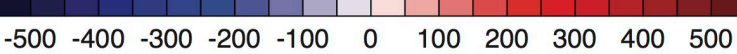

Figure S9. Southern and Northern hemispheres late winter maximal mixed layer depth difference $(\mathrm{m})$ between the sensitivity experiments and the Maastrichtian simulation. 
Deep Labrador Seaway - Maastrichtian
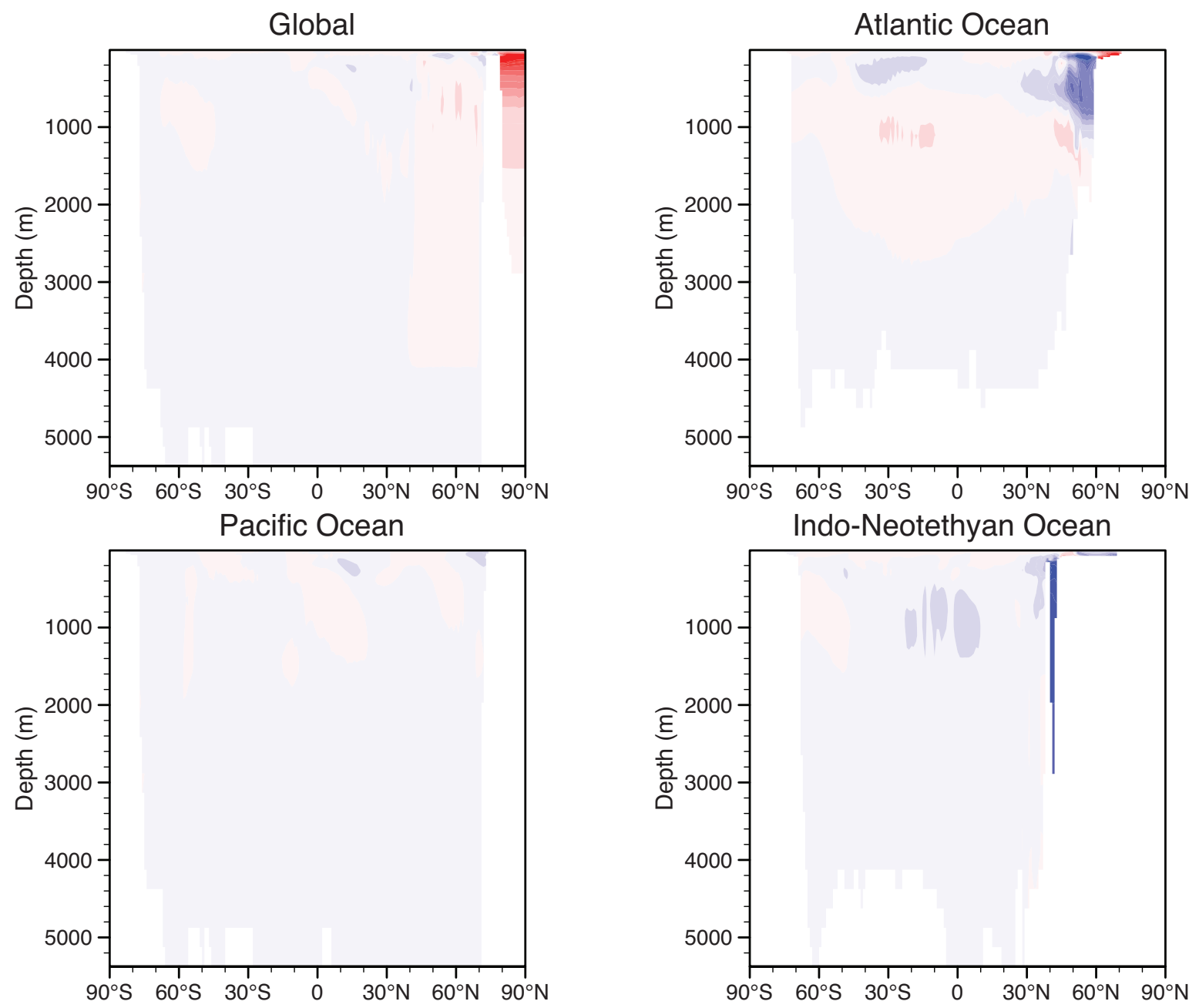

Temperature difference $\left({ }^{\circ} \mathrm{C}\right)$

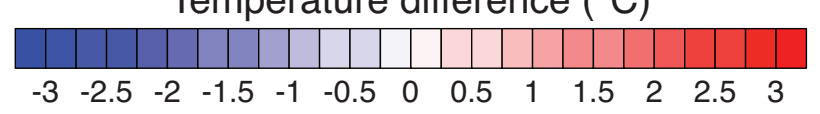

Figure S10. Zonally averaged ocean temperature changes $\left({ }^{\circ} \mathrm{C}\right)$ in the different basins between the Deep Labrador Seaway and the Maastrichtian simulations. 
A.

Maastrichtian

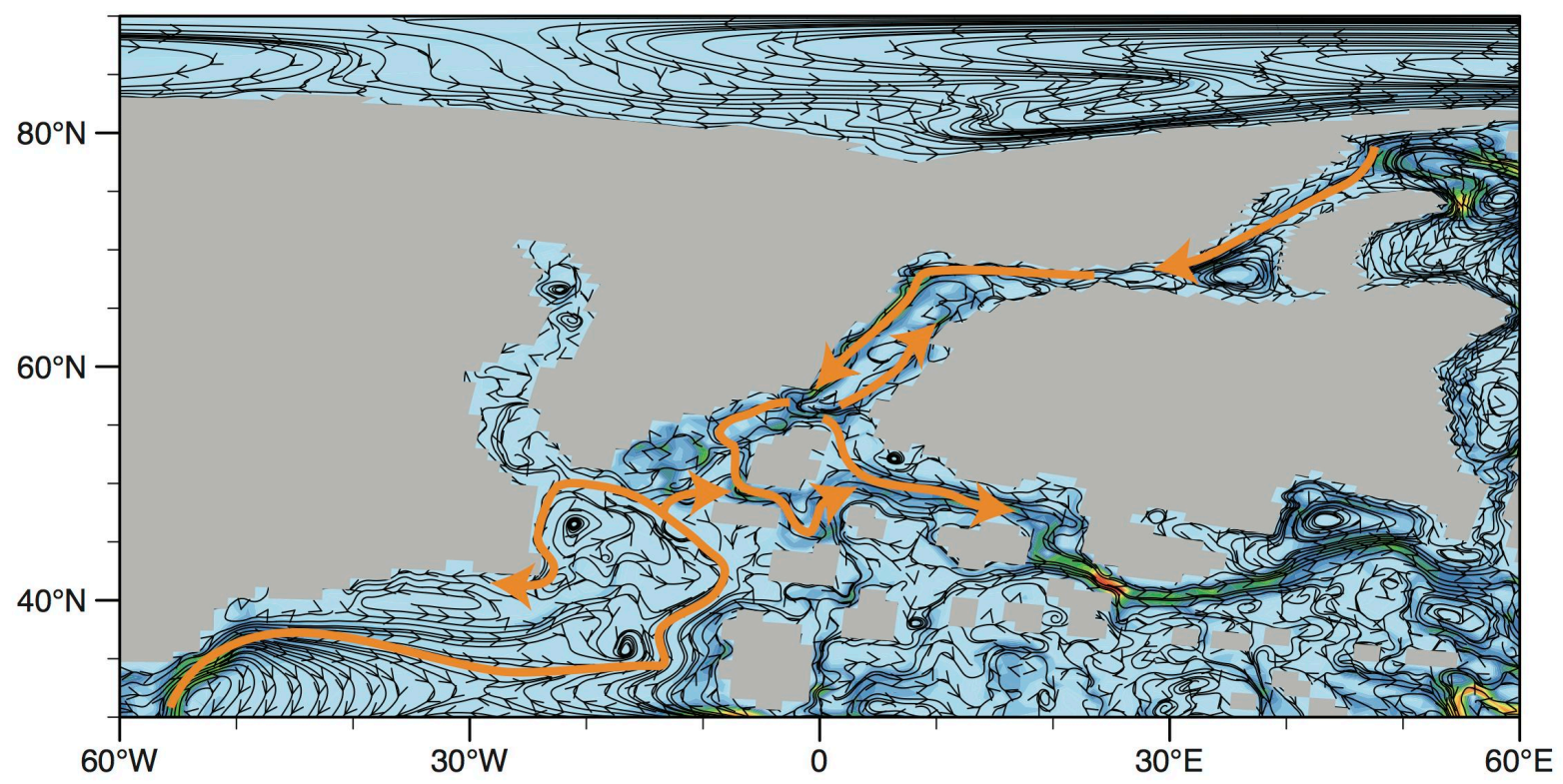

B.

Deep Labrador Seaway

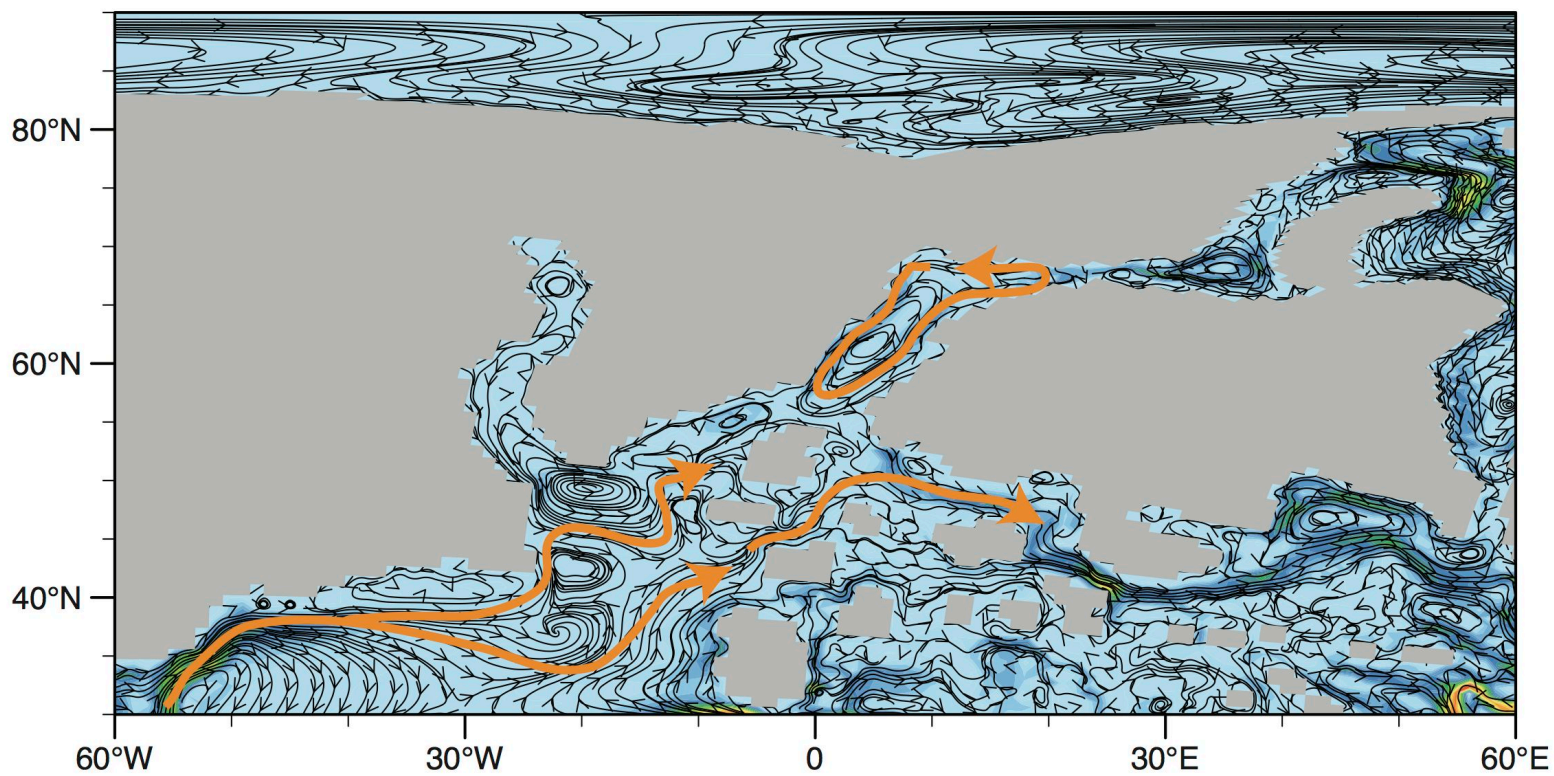

0-100 m depth ocean current intensity $\left(\mathrm{cm}^{\mathrm{s}} \mathrm{s}^{-1}\right)$

$\begin{array}{lllllllllllll}0 & 2 & 4 & 6 & 8 & 10 & 12 & 14 & 16 & 18 & 20 & 22 & 24\end{array}$

Figure S11. North Atlantic and northern Neotethys upper ocean circulation in (A) the Maastrichtian and (B) the Deep Labrador Seaway experiments. Orange arrows represent major current systems. 


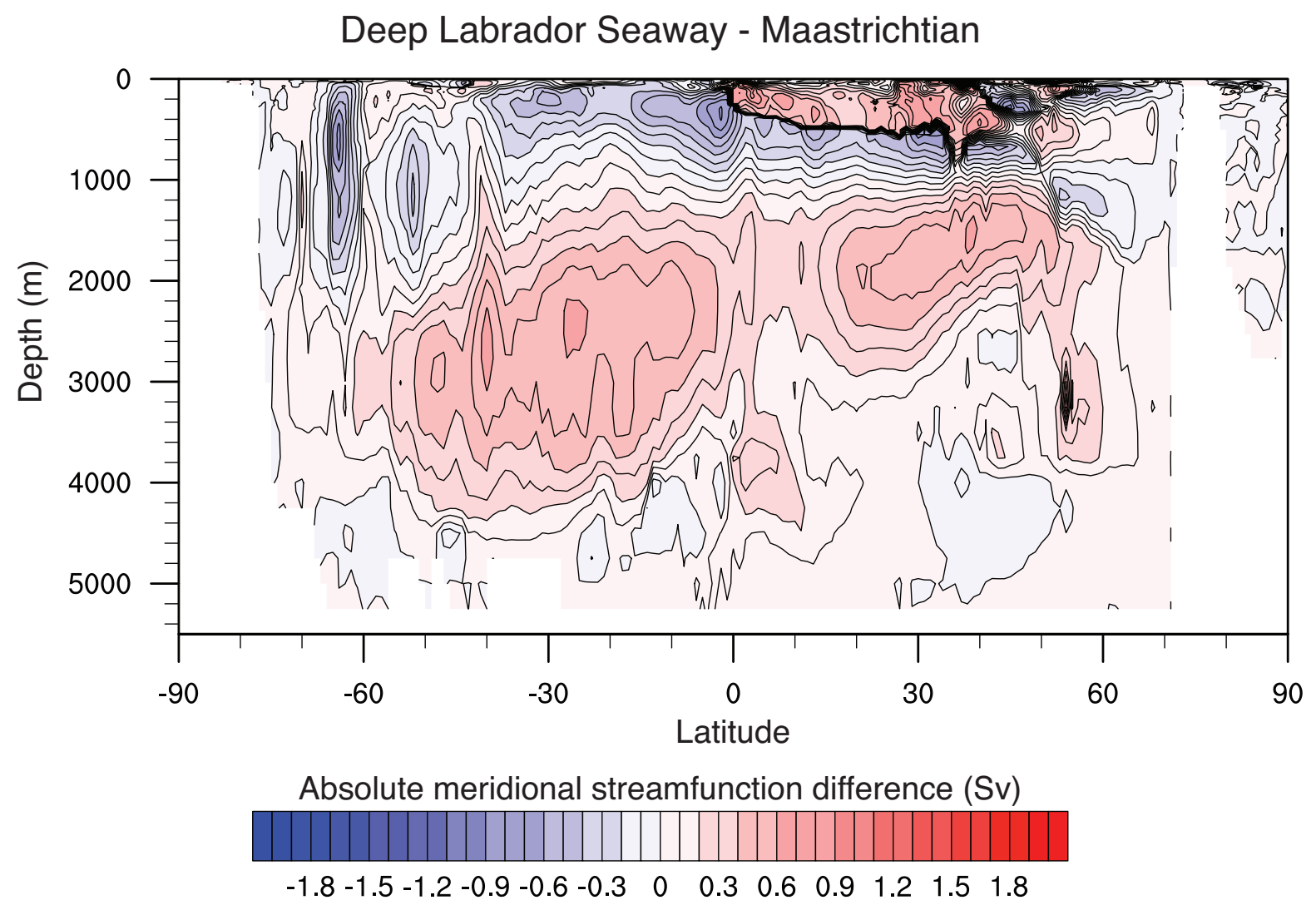

Figure S12. Difference of the absolute values of the global northward meridional streamfunction between the Deep Labrador Seaway and the Maastrichtian experiments. Positive values indicate a more vigorous circulation regardless of the sign of the global streamfunction in each simulation because the two global streamfunctions are very similar (Fig. 4B and 4C). 


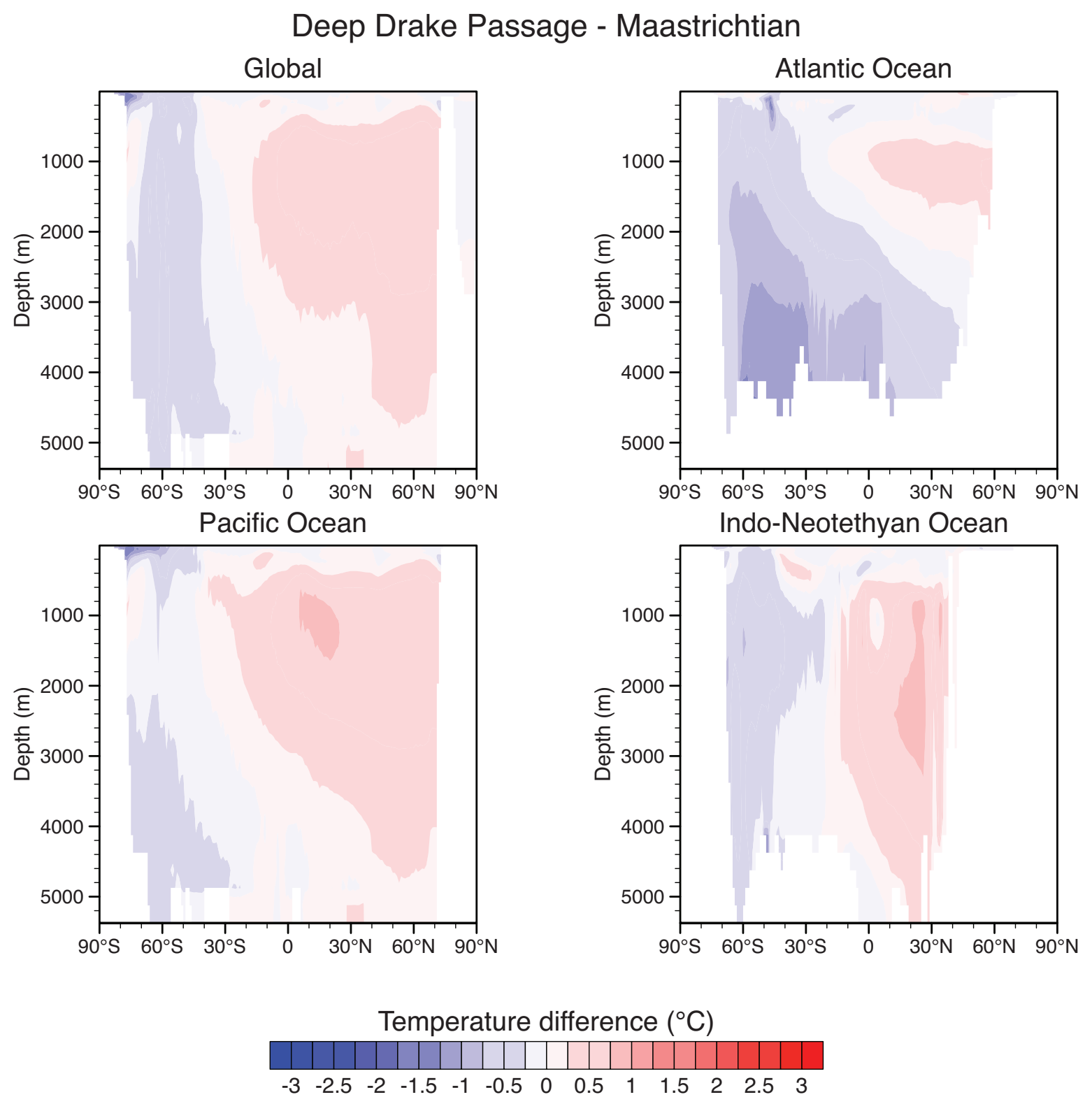

Figure S13. Zonally averaged ocean temperature changes $\left({ }^{\circ} \mathrm{C}\right)$ in the different basins between the Deep Drake Passage and the Maastrichtian simulations. 
Water flux across the Indonesian section
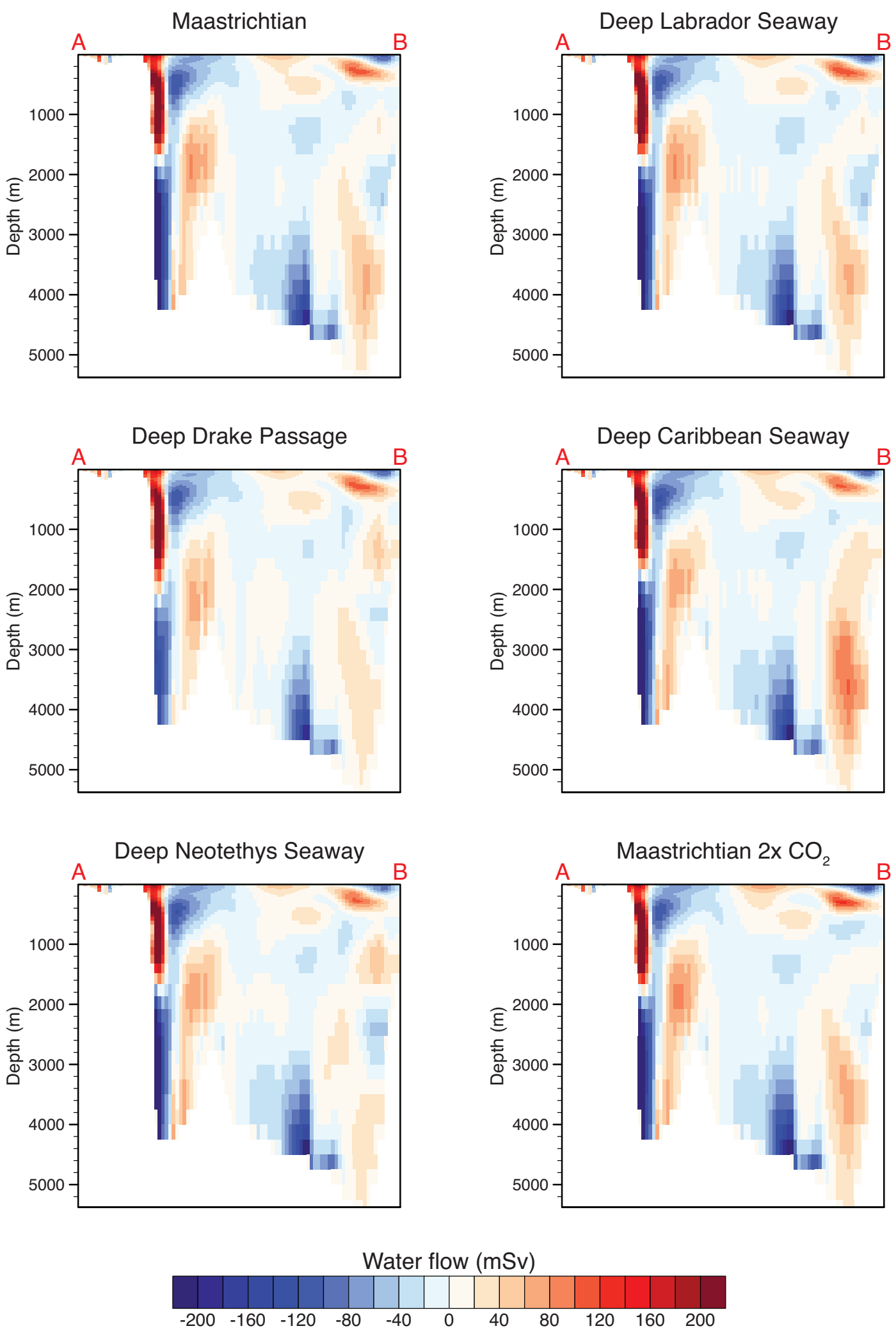

Figure S14. Water flux across the Indonesian section $(\mathrm{mSv})$ for the Maastrichtian and Maastrichtian sensitivity experiments. 

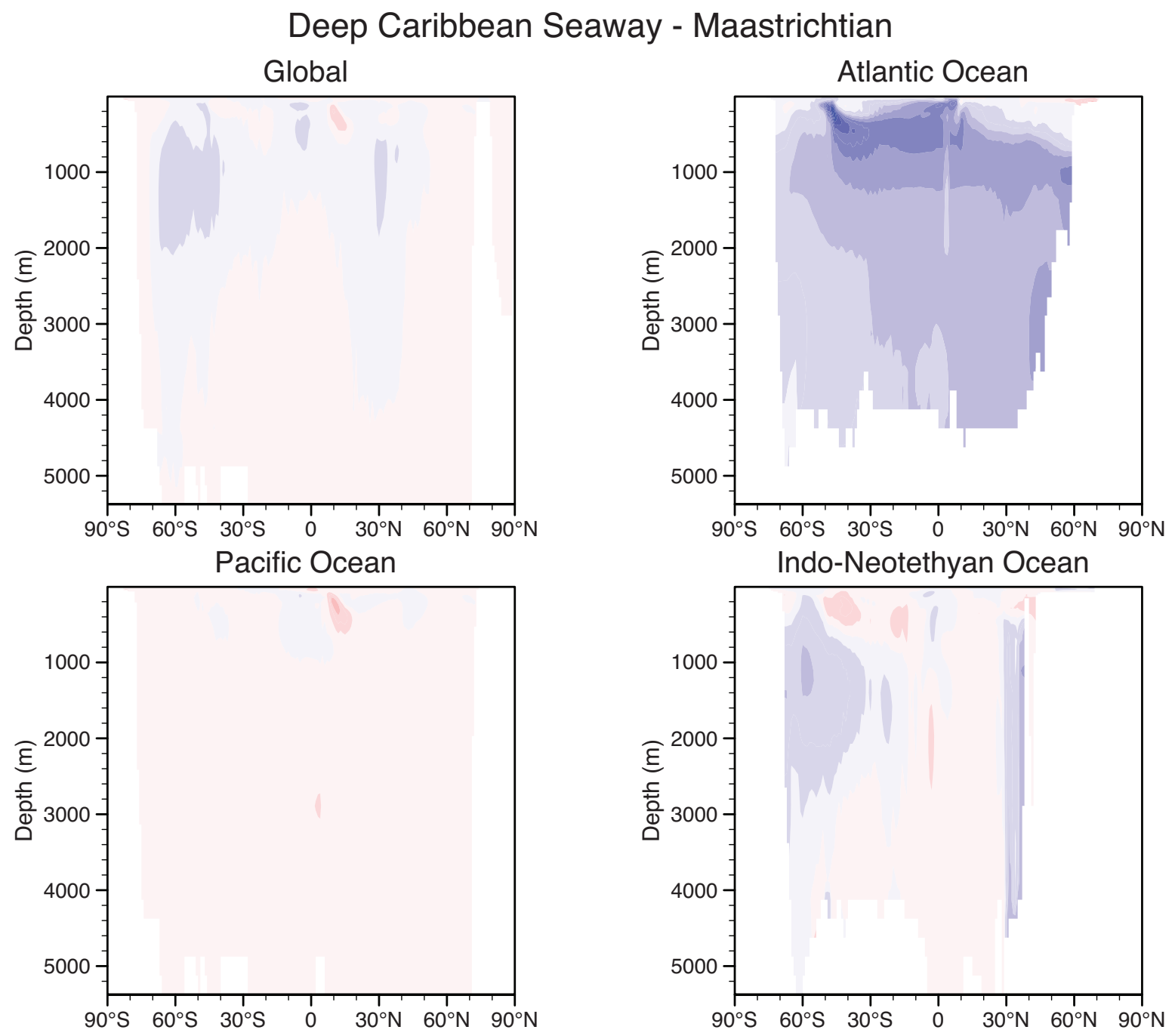

Temperature difference $\left({ }^{\circ} \mathrm{C}\right)$

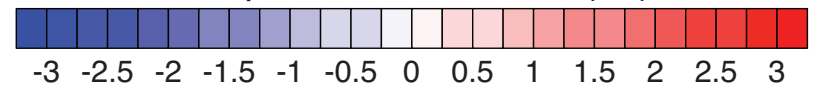

Figure S15. Zonally averaged ocean temperature changes $\left({ }^{\circ} \mathrm{C}\right)$ in the different basins between the Deep Caribbean Seaway and the Maastrichtian simulations. 


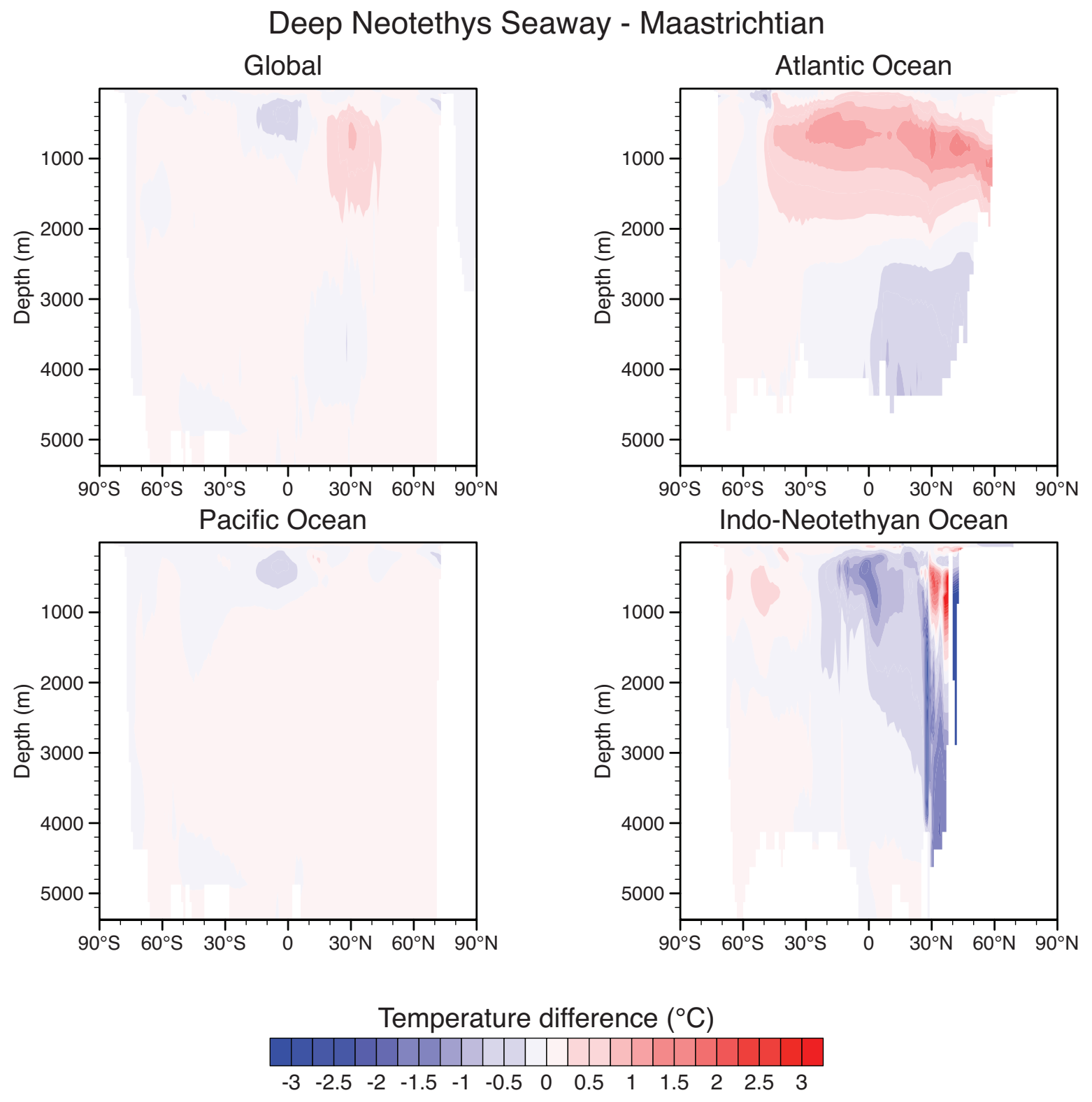

Figure S16. Zonally averaged ocean temperature changes $\left({ }^{\circ} \mathrm{C}\right)$ in the different basins between the Deep Neotethys Seaway and the Maastrichtian simulations. 
A.

Maastrichtian

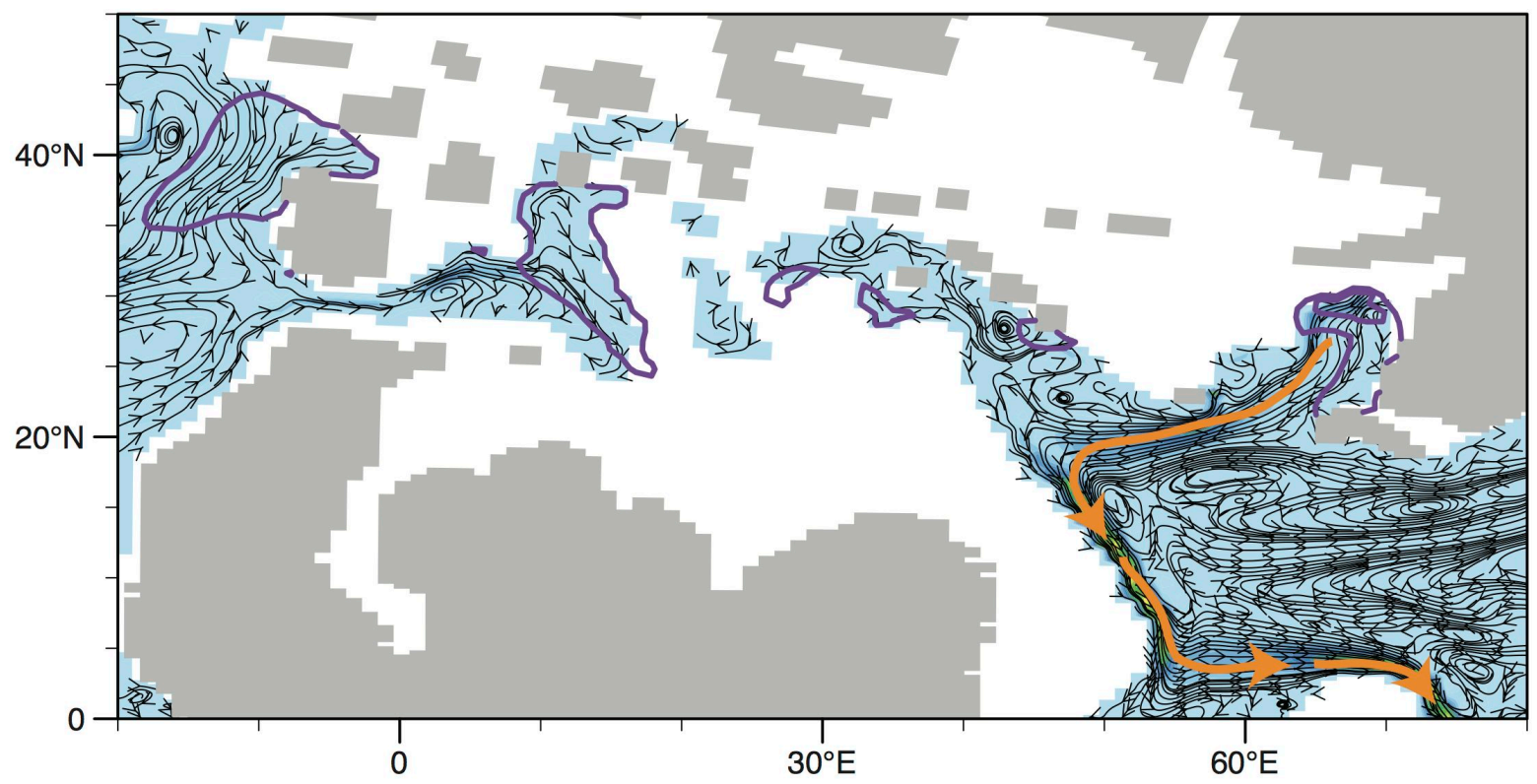

B. Deep Neotethys Seaway

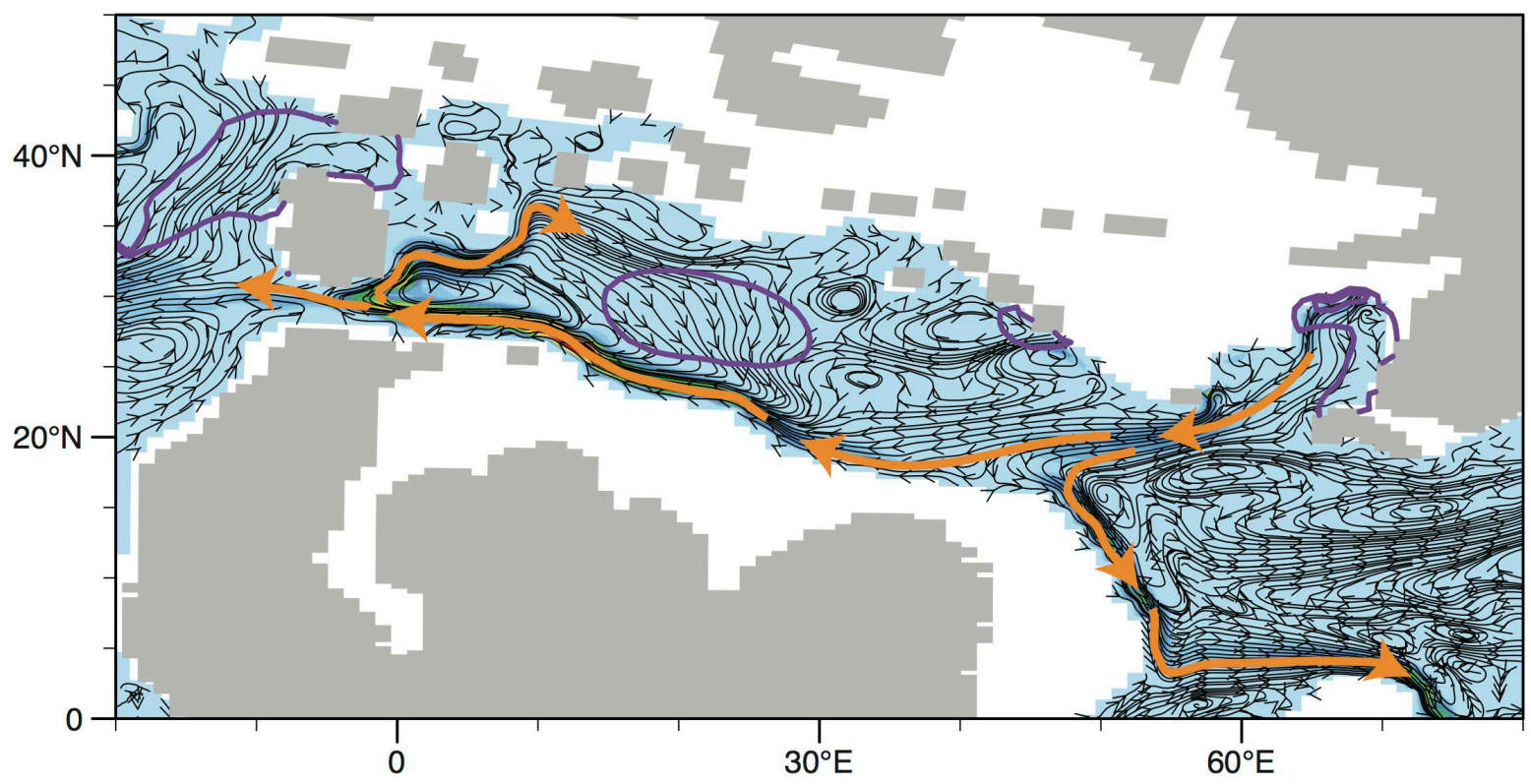

$500 \mathrm{~m}$ depth ocean current intensity $\left(\mathrm{cm} . \mathrm{s}^{-1}\right)$

$\begin{array}{lllllllllllll}0 & 1 & 2 & 3 & 4 & 5 & 6 & 7 & 8 & 9 & 10 & 11 & 12\end{array}$

Figure S17. Neotethys upper intermediate $(\sim 500 \mathrm{~m})$ ocean circulation in (A) the Maastrichtian and (B) the Deep Neotethys Seaway experiments. Orange arrows represent major current systems. Purple contours represent regions of late winter deepening of the mixed layer (200 m contours). 
A.

Maastrichtian

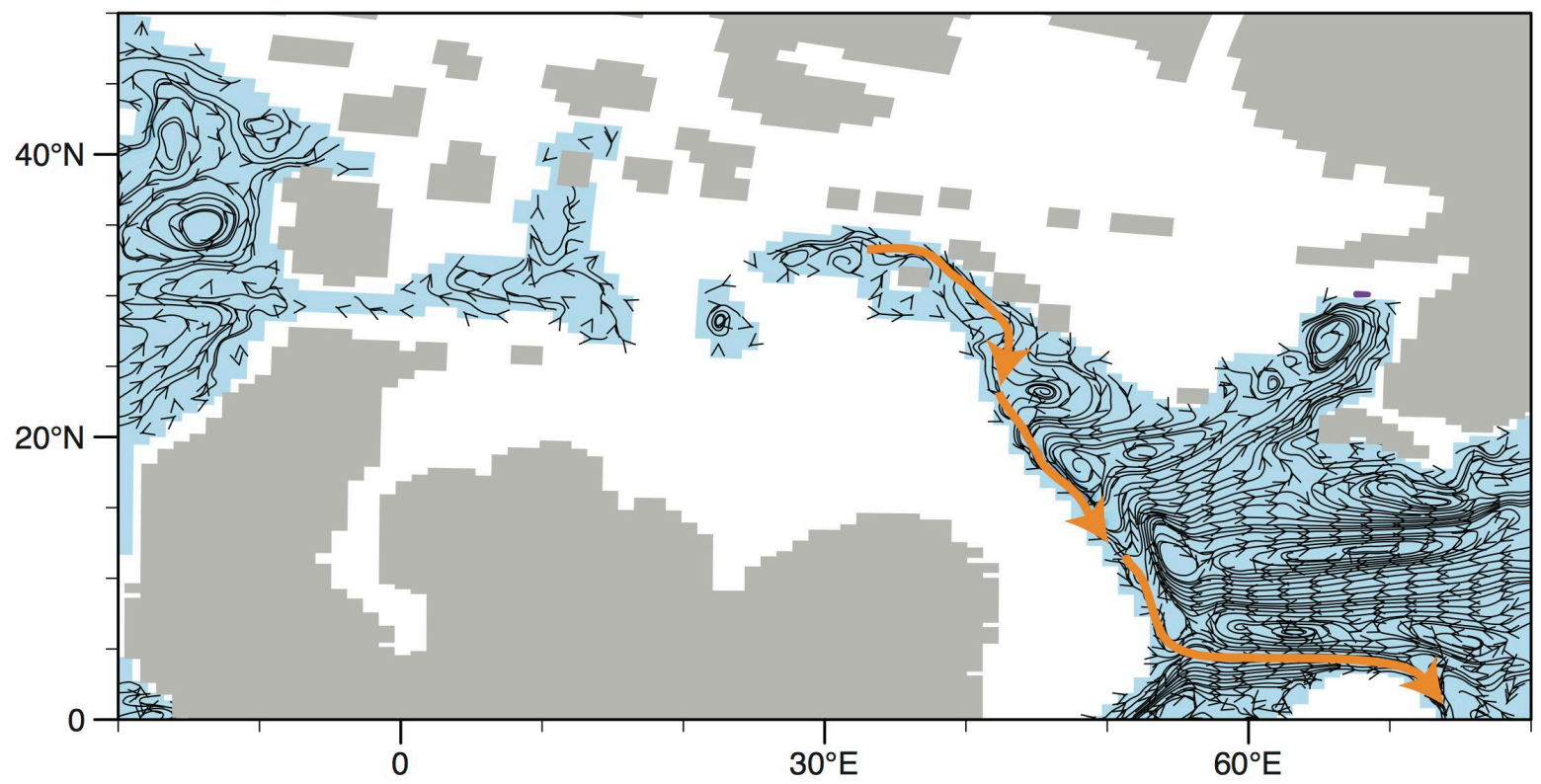

B. Deep Neotethys Seaway

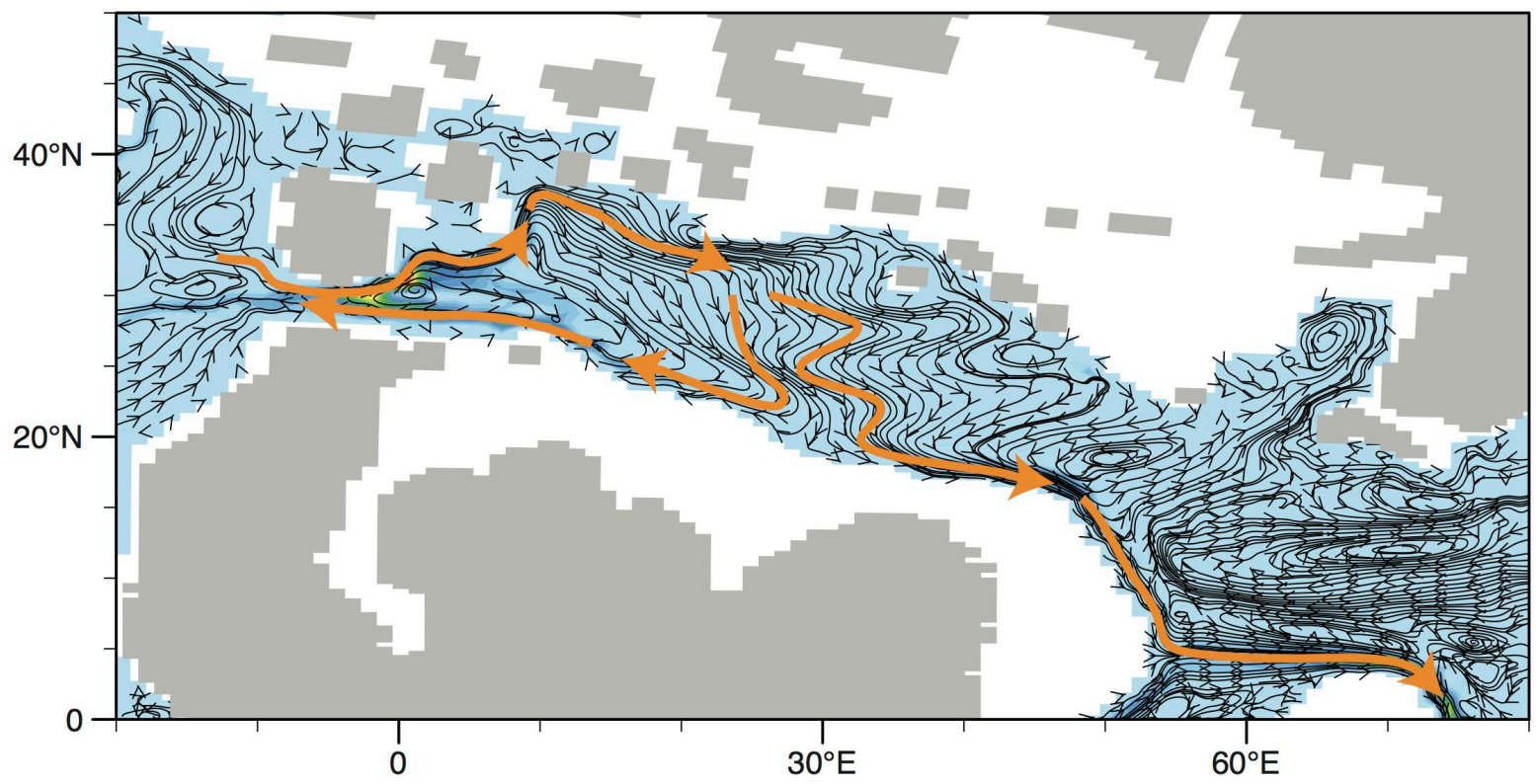

$1000 \mathrm{~m}$ depth ocean current intensity $\left(\mathrm{cm} \cdot \mathrm{s}^{-1}\right)$

$\begin{array}{lllllllllllll}0 & 0.5 & 1 & 1.5 & 2 & 2.5 & 3 & 3.5 & 4 & 4.5 & 5 & 5.5 & 6\end{array}$

Figure S18. Neotethys deep intermediate $(\sim 1000 \mathrm{~m})$ ocean circulation in (A) the Maastrichtian and (B) the Deep Neotethys Seaway experiments. Orange arrows represent major current systems. 


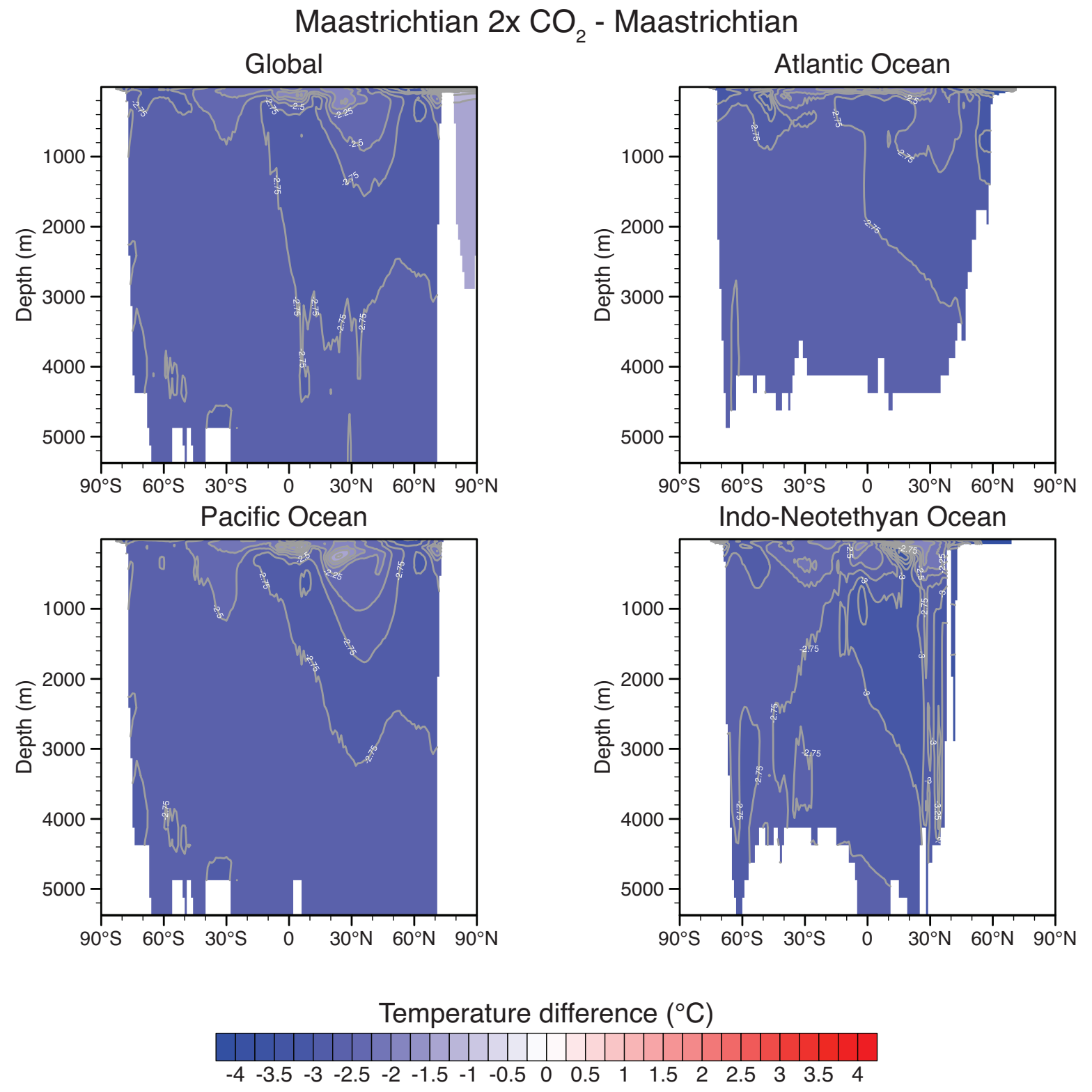

Figure S19. Zonally averaged ocean temperature changes $\left({ }^{\circ} \mathrm{C}, 0.25{ }^{\circ} \mathrm{C}\right.$ grey contours) in the different basins between the $2 \mathrm{x} \mathrm{CO}_{2}$ and the Maastrichtian simulations. 

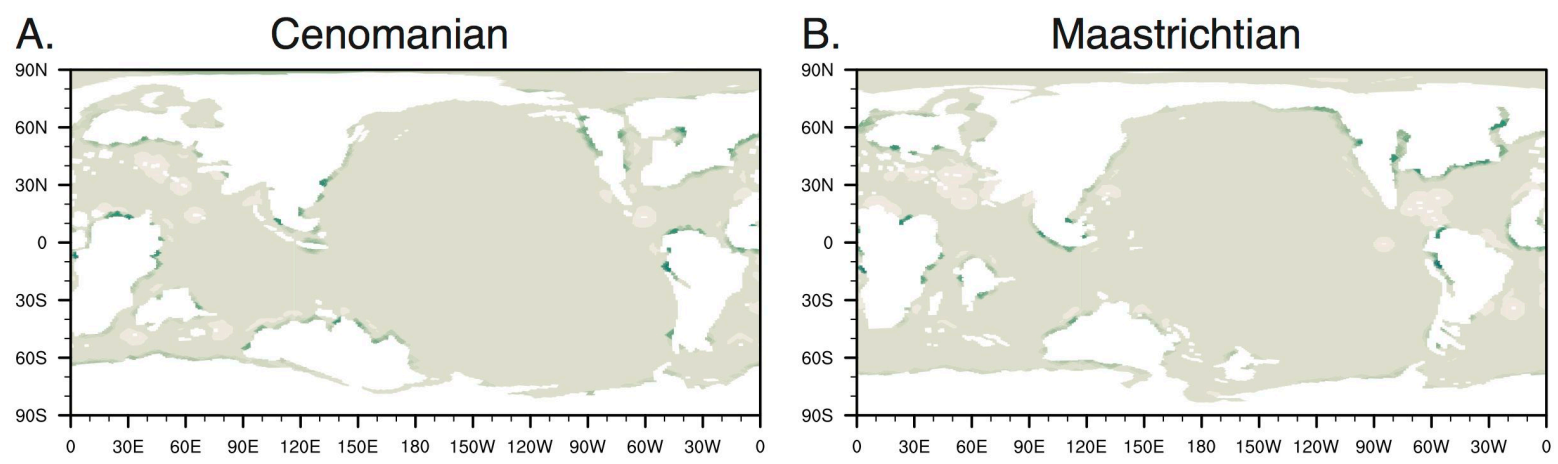

Annual mean runoff freshwater flux (mSv)

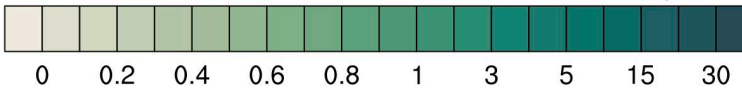

C.

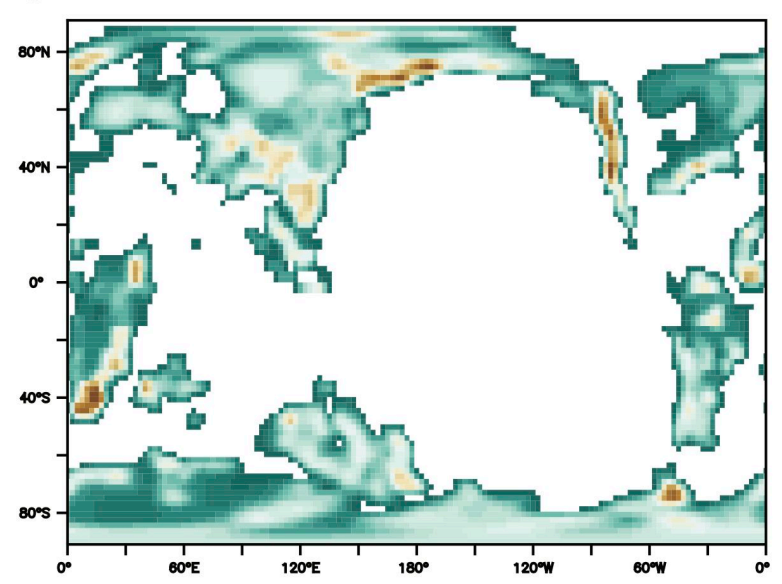

D.

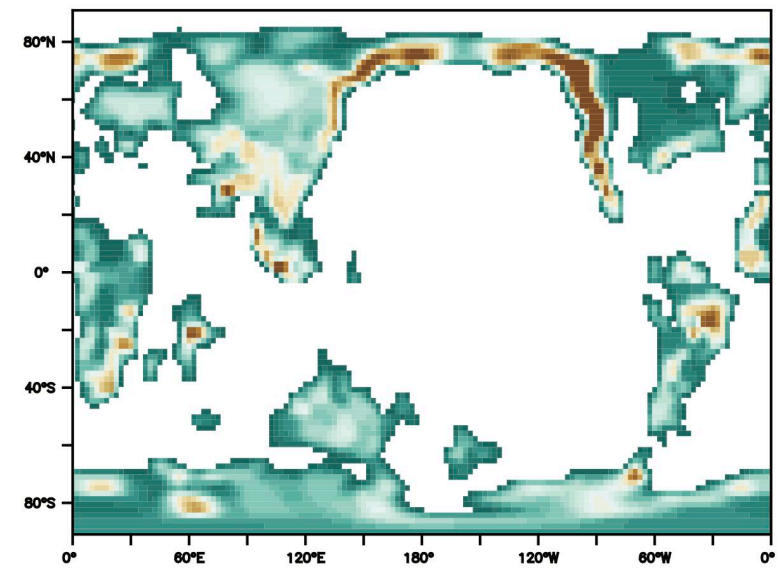

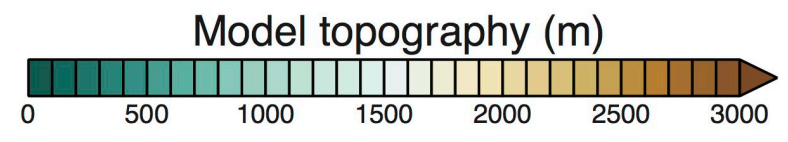

Figure S20. Annual mean runoff freshwater discharge into the ocean $(\mathrm{mSv})$ for $(\mathrm{A})$ the Cenomanian and (B) the Maastrichtian simulations. (C) Cenomanian topography seen by the CAM4 atmospheric model. (D) Same for the Maastrichtian. 
67 Doubrovine, P. V., and Tarduno, J. A.: Late Cretaceous paleolatitude of the Hawaiian hot 68 spot: New paleomagnetic data from Detroit Seamount (ODP Site 883), Geochemistry, 69 Geophysics, Geosystems, 5, 2004.

70 Frank, T. D., Thomas, D. J., Leckie, R. M., Arthur, M. A., Bown, P. R., Jones, K., and Lees, J.

71 A.: The Maastrichtian record from Shatsky Rise (northwest Pacific): A tropical perspective on global ecological and oceanographic changes, Paleoceanography, 20, 2005.

Gradstein, F. M., and Ludden, J. N.: 43. Legs 122 and 123, northwestern Australian margin-a stratigraphic and paleogeographic summary, Proceedings of the Ocean Drilling Program, Scientific Results, 1992, 801-816, Hague, A. M., Thomas, D. J., Huber, M., Korty, R., Woodard, S. C., and Jones, L. B.: Convection of North Pacific deep water during the early Cenozoic, Geology, 40, 527-530, 10.1130/g32886.1, 2012.

Haynes, S. J., MacLeod, K. G., Ladant, J. B., Vande Guchte, A., Rostami, M. A., Poulsen, C. J., \& Martin, E. E.: Constraining sources and relative flow rates of bottom waters in the Late Cretaceous Pacific Ocean, Geology, 2020.

Jiménez Berrocoso, Á., MacLeod, K. G., Martin, E. E., Bourbon, E., Londoño, C. I., and Basak, C.: Nutrient trap for Late Cretaceous organic-rich black shales in the tropical North Atlantic, Geology, 38, 1111-1114, 2010.

Le Houedec, S., Meynadier, L., Cogné, J. P., Allègre, C. J., and Gourlan, A. T.: Oceanwide imprint of large tectonic and oceanic events on seawater $\mathrm{Nd}$ isotope composition in the Indian Ocean from 90 to $40 \mathrm{Ma}$, Geochemistry, Geophysics, Geosystems, 13, 2012. MacLeod, K. G., Martin, E. E., and Blair, S. W.: Nd isotopic excursion across Cretaceous ocean anoxic event 2 (Cenomanian-Turonian) in the tropical North Atlantic, Geology, 36, 811-814, 2008.

MacLeod, K. G., Londoño, C. I., Martin, E. E., Berrocoso, Á. J., and Basak, C.: Changes in North Atlantic circulation at the end of the Cretaceous greenhouse interval, Nature Geoscience, 4, 779, 2011.

Martin, E. E., MacLeod, K. G., Berrocoso, A. J., and Bourbon, E.: Water mass circulation on Demerara Rise during the Late Cretaceous based on Nd isotopes, Earth and Planetary Science Letters, 327, 111-120, 2012.

Masson, D. G., Montadert, L., and Scrutton, R. A.: Regional Geology of the Goban Spur continental margin, Initial Reports of the Deep Sea Drilling Project, 80, 1115-1139, 1985. Moiroud, M., Pucéat, E., Donnadieu, Y., Bayon, G., Guiraud, M., Voigt, S., Deconinck, J.-F., and Monna, F.: Evolution of neodymium isotopic signature of seawater during the Late Cretaceous: Implications for intermediate and deep circulation, Gondwana Research, 36, 503-522, 2016.

Murphy, D. P., and Thomas, D. J.: Cretaceous deep-water formation in the Indian sector of the Southern Ocean, Paleoceanography, 27, PA1211, 10.1029/2011pa002198, 2012. Murphy, D. P., and Thomas, D. J.: The evolution of Late Cretaceous deep - ocean circulation in the Atlantic basins: Neodymium isotope evidence from South Atlantic drill sites for tectonic controls, Geochemistry, Geophysics, Geosystems, 14, 5323-5340, 2013. Robinson, S. A., Murphy, D. P., Vance, D., and Thomas, D. J.: Formation of "Southern $111874,10.1130 / \mathrm{g} 31165.1,2010$. 
112 Robinson, S. A., and Vance, D.: Widespread and synchronous change in deep-ocean

113 circulation in the North and South Atlantic during the Late Cretaceous,

114 Paleoceanography, 27, n/a-n/a, 10.1029/2011pa002240, 2012.

115 Thomas, D. J.: Evidence for deep-water production in the North Pacific Ocean during the 116 early Cenozoic warm interval, Nature, 430, 65, 2004.

117 Thomas, D. J., Korty, R., Huber, M., Schubert, J. A., and Haines, B.: Nd isotopic structure of 118 the Pacific Ocean 70-30 Ma and numerical evidence for vigorous ocean circulation and 119 ocean heat transport in a greenhouse world, Paleoceanography, 29, 454-469, 2014.

120 Voigt, S., Jung, C., Friedrich, O., Frank, M., Teschner, C., and Hoffmann, J.: Tectonically 121 restricted deep-ocean circulation at the end of the Cretaceous greenhouse, Earth and 122 Planetary Science Letters, 369, 169-177, 2013. 